ESAIM: M2AN 50 (2016) 541-563

DOI: $10.1051 / \mathrm{m} 2 \mathrm{an} / 2015054$
ESAIM: Mathematical Modelling and Numerical Analysis

www.esaim-m2an.org

\title{
NITSCHE'S METHOD FOR PARABOLIC PARTIAL DIFFERENTIAL EQUATIONS WITH MIXED TIME VARYING BOUNDARY CONDITIONS
}

\author{
Anna Tagliabue ${ }^{1}$, Luca Dedì ${ }^{2}$ And Alfio Quarteroni ${ }^{1,2}$
}

\begin{abstract}
We investigate a finite element approximation of an initial boundary value problem associated with parabolic Partial Differential Equations endowed with mixed time varying boundary conditions, switching from essential to natural and vice versa. The switching occurs both in time and in different portions of the boundary. For this problem, we apply and extend the Nitsche's method presented in [Juntunen and Stenberg, Math. Comput. (2009)] to the case of mixed time varying boundary conditions. After proving existence and numerical stability of the full discrete numerical solution obtained by using the $\theta$-method for time discretization, we present and discuss a numerical test that compares our method to a standard approach based on remeshing and projection procedures.
\end{abstract}

Mathematics Subject Classification. 35K20, 65M12, 65M60, 68U20, 74S05.

Received May 6, 2015. Revised July 9, 2015.

Published online March 14, 2016.

\section{INTRODUCTION AND MOTIVATION}

The study of initial boundary value problems associated with parabolic Partial Differential Equations (PDEs) and complemented with mixed time varying boundary conditions (BCs) represents a challenging task both for the theoretical analysis and the numerical approximation. This kind of model may be suitable to describe physical problems as, e.g. the distribution of the temperature in a body with temperature and heat flux prescribed on a portion of the boundary changing in time, or flows in cavities (e.g. the heart ventricles and atria) with opening and closing valves. Despite its applicative interest, to the best of our knowledge, neither numerical examples nor numerical studies are available in literature. On the other hand, in the last decades, several works have been devoted to the analysis of this class of problems, even if only from a theoretical point of view $[1,5,9,10,18,21,29]$. In particular, the focus has been on the existence and regularity properties of the solution; in this respect, since the 1970s, different techniques have been developed. Starting from the standard theory of abstract evolution equations of Kato, Lions, and Magens [18,19], works as [9,10] adapted these arguments to the case of time varying mixed BCs, leading to solutions with properties connected to the geometrical structure of the boundaries. Then, by developing a more general result on abstract evolution equations in variable domains, Baiocchi [5] investigated

Keywords and phrases. Nitsche's method, parabolic problems, mixed time varying boundary conditions, stability analysis, finite element method.

1 MOX - Modeling and Scientific Computing, Mathematics Department "F. Brioschi", Politecnico di Milano, via Bonardi 9, 20133 Milano, Italy. anna.tagliabue@polimi.it

2 CMCS - Chair of Modeling and Scientific Computing, MATHICSE - Mathematics Institute of Computational Science and Engineering, EPFL - École Polytechnique Fédérale de Lausanne, Station 8, 1015 Lausanne, Switzerland 
the solution of these problems in spaces independent of the geometry under relatively weak assumptions on the data and on the regularity of the boundary. This latter approach has been further extended by Savaré in [29].

In [29], it is noted that the proposed abstract approach for the proof of the existence of the solution of a parabolic PDE defined by a second order elliptic spatial operator and endowed with mixed time varying BCs can be extended to a Finite Element (FE) formulation [25]. Starting from the framework of [29], one can consider a numerical method based on the backward Euler (BE) method [25] for the discretization in time and on the FE method for the spatial approximation. Specifically, one can use the standard (strong) imposition of Dirichlet BCs in the discretization of the parabolic PDE with mixed time varying BCs, thus building a family of closed time varying $\mathrm{FE}$ function subspaces. This method, which we identify with the name time varying FE method, exhibits some drawbacks which may considerably affect the computational performances of the simulations. Indeed, at each time step, the number of degrees of freedom associated to the FE space changes and, as well as, the size and the structure of the associated discrete problem. Moreover, when implementing the time varying FE method by using commercial codes, it may be necessary to re-mesh the domain and use extension and projection operators of the intermediate solutions for all the time steps.

In order to overcome the drawbacks of the time varying $\mathrm{FE}$ method, in this work we propose and analyse a new numerical approach for mixed time varying BCs which yields a FE semi-discretized spatial approximation with time independent function spaces and is based on the Nitsche's method [22]. Moreover, even if in this paper we focus on the $\theta$-method $[25,26]$ for the time discretization, we highlight that any other scheme could similarly be used. We remark that the Nitsche's method for parabolic problems with mixed time varying BCs is formulated in the framework of [17], but considering the more general case of space-time weighting functions in place of constant coefficients, characterizing both the type of data and the degree of penalization; in addition we consider a general advection-diffusion-reaction PDE. We remark that the proposed approach introduces penalty and consistency terms in the variational formulation of the problem. Specifically, the Dirichlet BCs are imposed weakly in the variational formulation rather than strongly in the space of test functions by means of penalty terms; for the Neumann BCs, additional terms are introduced with respect to those of the classical formulation.

In our theoretical analysis we prove the well posedness of the semi-discrete problem; then, we study the stability of the full discrete problem with stability conditions depending on both the data and the penalty functions. We show results in accordance with the ones presented in literature involving the weak imposition of essential BCs, which is commonly used in the context of the Discontinuous Galerkin (DG) method [25, 27]. As a matter of fact, the weak imposition of essential BCs (see e.g. [6]) represents a particular case of the more general scheme proposed in this paper.

This paper is organized as follows. In Section 2, we describe the continuous problem and we recall the results on the existence and regularity properties of the solution already established in [29]. Then, we present the standard numerical method based on the BE scheme for the time discretization and the spatial approximation by means of the FE method with time varying spaces in the framework of [29]. In Section 3, we introduce the proposed approach for mixed time varying BCs based on the Nitsche's method. We analyse the proposed semi-discrete formulation, then we introduce the approximation in time by using the $\theta$-method, and we finally analyse the fully discretized problem; specifically, we provide results on the numerical stability of the method. Finally, in Section 4, we report and discuss some numerical results to highlight the efficiency of the proposed method. Conclusions follow.

\section{The CONTINUOUS PROBLEM}

In this section, we introduce the parabolic problem with mixed time varying BCs. In Section 2.1 we recall some notions and notations on Banach spaces by referring in particular to [2], we describe the problem defined by a PDE with a second order elliptic spatial operator and we recall a result on the existence and uniqueness of the solution of Savaré in [29]. Finally, in Section 2.2, we present the FE method with time varying function spaces. 


\subsection{The continuous problem: Preliminaries and well-posedness analysis}

We assume that $\Omega \subseteq \mathbb{R}^{n}$, with $n \in \mathbb{N}$, is an open connected bounded set with regular boundary $\Gamma \equiv \partial \Omega$, satisfying a strong local Lipschitz condition. The independent spatial variable $\mathbf{x}$ will be defined in $\Omega$, while the independent time variable $t$ will take values in an interval $I:=(0, T) \subseteq \mathbb{R}$ with $0<T<\infty$. For any positive integer $m \geq 0$ and any real number $1 \leq p \leq \infty$, we denote the Sobolev space of order $m$ as $\mathrm{W}^{m, p}(\Omega):=$ $\left\{v \in \mathrm{L}^{p}(\Omega): \mathrm{D}^{(\iota)} v \in \mathrm{L}^{p}(\Omega)\right.$ for all $\iota=\left(\iota_{1}, \ldots, \iota_{d}\right) \in \mathbb{N}^{n}$ with $\left.0 \leq|\iota|=\iota_{1}+\ldots+\iota_{n} \leq m\right\}$, with $\mathrm{D}^{(\iota)}$ denoting the distributional partial differential operator. The corresponding norm and semi-norm are indicated as $\|\cdot\|_{\mathrm{W}^{m, p}(\Omega)}$ and $|\cdot|_{\mathrm{W}^{m, p}(\Omega)}$, respectively. Moreover, for $p=2$, we denote by $\mathrm{H}^{m}(\Omega)$ the Hilbert space $\mathrm{W}^{m, 2}(\Omega)$ and by $\mathrm{H}_{\Gamma_{D}}^{m}(\Omega)$ the space of functions $v \in \mathrm{H}^{m}(\Omega)$ vanishing on $\Gamma_{D} \subseteq \Gamma \equiv \partial \Omega$ in the sense of the traces, i.e. $\mathrm{H}_{\Gamma_{D}}^{m}:=\left\{v \in \mathrm{H}^{m}:\left.v\right|_{\Gamma_{D}} \equiv 0\right\}$.

Let $\mathcal{H}$ be a Banach space endowed with norm $\|\cdot\|_{\mathcal{H}}$ defined over $\Omega$. Then, the space of measurable functions with respect to the Lebesgue measure, defined on the interval $I$ and having values in $\mathcal{H}$ with finite Bochner integral on $I$, is denoted by $\mathrm{L}^{1}(I ; \mathcal{H}):=\left\{v: I \rightarrow \mathcal{H}: \int_{I}\|v(s)\|_{\mathcal{H}} d s<\infty\right\}$. More generally, for any $p$ such that $1 \leq p<\infty$, we have $L^{p}(I ; \mathcal{H}):=\left\{v: I \rightarrow \mathcal{H}: \int_{I}\|v(s)\|_{\mathcal{H}}^{p} d s<\infty\right\}$, and $\mathrm{L}^{\infty}(I ; \mathcal{H}):=\left\{v: I \rightarrow \mathcal{H}: \operatorname{ess}_{\sup _{t \in I}}\|v(t)\|_{\mathcal{H}}<\infty\right\}$. Analogously, for any positive integer $m \geq 0$, we consider the Hilbert spaces of measurable functions defined on $I$ with values in $\mathcal{H}$, say $\mathrm{H}^{m}(I ; \mathcal{H})=\left\{v \in \mathrm{L}^{2}(I ; \mathcal{H})\right.$ : $D_{t}^{(\iota)} v \in \mathrm{L}^{2}(I ; \mathcal{H})$ for all $\iota=\left(\iota_{1}, \ldots, \iota_{d}\right) \in \mathbb{N}^{d}$ with $\left.0 \leq|\iota|=\iota_{1}+\ldots+\iota_{d} \leq m\right\}$, where $D_{t}^{(\iota)}$ is the distributional partial derivative with respect to the independent variable $t \in I$. Finally, for any fractional numbers, $r$ and $s \in \mathbb{Q}$, we denote by $\mathrm{H}^{r, s}(\Omega)$ the space defined as $\mathrm{H}^{r, s}(\Omega)=\mathrm{L}^{2}\left(I ; \mathrm{H}^{r}(\Omega)\right) \cap \mathrm{H}^{s}\left(I ; \mathrm{L}^{2}(\Omega)\right)$.

Now, let $Q_{T}=\Omega \times(0, T)$ be the space-time domain. We consider the linear parabolic equation in the unknwn $u: Q_{T} \rightarrow \mathbb{R}$, which reads:

$$
\frac{\partial u}{\partial t}(\mathbf{x}, t)+\mathcal{L} u(\mathbf{x}, t)=f(\mathbf{x}, t) \quad \text { in } Q_{T},
$$

endowed with suitable initial and boundary conditions; $f: Q_{T} \rightarrow \mathbb{R}$ is the source term and $\mathcal{L}$ is a linear elliptic second order partial differential operator in the form:

$$
\mathcal{L} u=-\nabla \cdot(\sigma \nabla u)+\boldsymbol{\beta} \cdot \nabla u+\kappa u .
$$

Specifically, the diffusivity tensor $\boldsymbol{\sigma}$, with $\sigma_{i j} \in \mathrm{W}^{1, \infty}\left(Q_{T}\right)$, is assumed to be symmetric and elliptic, i.e. $\sigma_{i j}=\sigma_{j i}$ and:

$$
\exists \alpha>0: \sum_{i, j=1}^{n} \sigma_{i j}(\mathbf{x}, t) \xi_{i} \xi_{j} \geq \alpha|\xi|^{2} \quad \forall \xi \in \mathbb{R}^{n}, \forall(\mathbf{x}, t) \in Q_{T} ;
$$

moreover, we require a global Lipschitz condition on the coefficients $\sigma_{i j}$ for any $i, j=1, \ldots, n$. The vector $\boldsymbol{\beta}$ is supposed to be solenoidal $(\nabla \cdot \boldsymbol{\beta}=0)$ with components $\beta_{i} \in \mathrm{L}^{\infty}\left(Q_{T}\right)$; finally, $\kappa \in \mathrm{L}^{\infty}\left(Q_{T}\right)$ represents the reaction term. We highlight that, under the hypothesis on $\boldsymbol{\beta}, \mathcal{L} u$ can be rewritten as $\mathcal{L} u=-\nabla \cdot(\boldsymbol{\sigma} \nabla u-u \boldsymbol{\beta})+\kappa u$.

In order to define the mixed time varying boundary value Cauchy problem associated with equation (2.1), we introduce, analogously to [29], a family of $\mathrm{C}^{1,1}$ submanifolds with boundary $\Gamma_{D}^{(t)}$ for $t \in(0, T)$ on the lateral boundary $\Sigma=\Gamma \times(0, T)$. We denote by $\Sigma_{D}$ and $\Sigma_{N}$ the subsets of $\Sigma$ covered by these submanifolds and their complement defined as:

$$
\Sigma_{D}:=\bigcup_{t \in(0, T)} \Gamma_{D}^{(t)} \times\{t\} \quad \text { and } \quad \Sigma_{N}:=\Sigma \backslash \overline{\Sigma_{D}}
$$

respectively; $\Sigma_{D}$ and $\Sigma_{N}$ represent the lateral time varying parts of the boundary on which we prescribe Dirichlet and Neumann BCs as:

$$
\begin{array}{ll}
u(\mathbf{x}, t)=g(\mathbf{x}, t) & \text { on } \Sigma_{D} \\
-\boldsymbol{\Phi}_{\mathrm{diff}}(u(\mathbf{x}, t)) \cdot \mathbf{n}=G(\mathbf{x}, t) & \text { on } \Sigma_{N}
\end{array}
$$


respectively, where $\mathbf{n}$ indicates the outward directed unit vector normal to $\Sigma_{N}$, while $\boldsymbol{\Phi}_{\text {diff }}$ denotes the diffusive flux tensor associated to $\mathcal{L}$, which reads $\boldsymbol{\Phi}_{\text {diff }}(u):=-\sigma \nabla u$. We suppose that the functions $g: \Sigma_{D} \rightarrow \mathbb{R}$ and $G: \Sigma_{N} \rightarrow \mathbb{R}$ are defined in suitable trace spaces. Finally, the problem (2.1) endowed with mixed time varying BCs and initial condition $u_{0}: \Omega \rightarrow \mathbb{R}$ reads:

$$
\text { find } u: Q_{T} \rightarrow \mathbb{R}: \begin{cases}\frac{\partial u}{\partial t}(\mathbf{x}, t)+\mathcal{L} u(\mathbf{x}, t)=f(\mathbf{x}, t) & \text { in } \Omega \times(0, T), \\ u(\mathbf{x}, t)=g(\mathbf{x}, t) & \text { on } \Sigma_{D}, \\ -\boldsymbol{\Phi}_{\mathrm{diff}}(u(\mathbf{x}, t)) \cdot \mathbf{n}=G(\mathbf{x}, t) & \text { on } \Sigma_{N} . \\ u(\mathbf{x}, 0)=u_{0}(\mathbf{x}), & \text { in } \Omega .\end{cases}
$$

We recall a result on the existence and uniqueness of the solution of equation (2.6) provided by Savaré in [29]. ${ }^{3}$ Specifically, by introducing the distance $d(\cdot, \cdot)$ defining a metric space over $\Sigma_{D}$, we focus on the case in which a two-sided condition on the dilatation of the Dirichlet boundary $\Gamma_{D}^{(t)}$ is defined by the Hausdorff distance as:

$$
d_{H}\left(\Gamma_{D}^{(t)}, \Gamma_{D}^{(s)}\right)=\max \left\{\sup _{\mathbf{y} \in \Gamma_{D}^{(s)}} \inf _{\mathbf{x} \in \Gamma_{D}^{(t)}} d(\mathbf{x}, \mathbf{y}), \sup _{\mathbf{x} \in \Gamma_{D}^{(t)}} \inf _{\mathbf{y} \in \Gamma_{D}^{(s)}} d(\mathbf{x}, \mathbf{y})\right\}
$$

Theorem 2.1. Let the source term $f \in \mathrm{L}^{2}\left(Q_{T}\right)$, the initial condition $u_{0} \in \mathrm{H}^{1}(\Omega)$, and the boundary data $g \in \mathrm{H}^{3 / 2,3 / 4}\left(\Sigma_{D}\right)$ and $G \in \mathrm{H}^{1 / 2,1 / 4}\left(\Sigma_{N}\right)$, with the initial compatibility condition $u_{0}(\mathbf{x})=g(\mathbf{x}, 0)$ on $\Gamma_{D}^{(0)}$ satisfied. If the excess of dilatation $d_{H}\left(\Gamma_{D}^{(t)}, \Gamma_{D}^{(s)}\right)$ of equation $(2.7)$ is controlled by a weighted measure of the interval of time, i.e.:

$$
\exists \rho \in \mathrm{L}^{4}(0, T) \quad: \quad d_{H}\left(\Gamma_{D}^{(t)}, \Gamma_{D}^{(s)}\right) \leq \int_{s}^{t} \rho(\tau) \mathrm{d} \tau, \quad 0 \leq s<t \leq T,
$$

then, equation (2.6) possesses a unique solution $u: Q_{T} \rightarrow \mathbb{R}$ such that:

$$
\frac{\partial u}{\partial t}, \mathcal{L} u \in \mathrm{L}^{2}\left(Q_{T}\right), \text { and } u \in \mathrm{C}^{0}\left(0, T ; \mathrm{H}^{1}(\Omega)\right) .
$$

\subsection{FE method with time varying function spaces}

In order to introduce a spatial FE approximation of the PDE (2.6), firstly, we recast this problem in weak formulation. For any $t \in(0, T)$, we define the trial affine space $V_{g}^{(t)}:=\left\{v \in \mathrm{H}^{1}(\Omega):\left.v(t, \mathbf{x})\right|_{\Gamma_{D}^{(t)}}=g(t, \mathbf{x})\right\}$ and we introduce a lifting function $\bar{g}(t, \mathbf{x}) \in \mathrm{H}^{1}(\Omega)$ satisfying the essential BC (2.6b) in the sense of the traces, i.e. such that $\gamma_{\Gamma_{D}^{(t)}} \bar{g}(t, \mathbf{x})=g(t, \mathbf{x})$. Moreover, $\bar{g}$ is such that for all the functions $u \in V_{g}^{(t)}$ there exists a unique $w \in V_{0}^{(t)}:=\left\{v \in \mathrm{H}^{1}(\Omega):\left.v(t, \mathbf{x})\right|_{\Gamma_{D}^{(t)}}=0\right\}$ for which $u=\bar{g}+w$. We remark that, the Dirichlet BC (2.6b) is imposed strongly in the space of test functions, while the Neumann BC (2.6c) is weakly introduced in the variational formulation. The weak formulation of equation (2.6) reads for a.e. $t \in(0, T)$ :

$$
\text { find } w(t) \in V_{0}^{(t)}: \quad\left(\frac{\partial w}{\partial t}(t), \varphi\right)+a_{\boldsymbol{\sigma}, \boldsymbol{\beta}, \kappa}(t ; w(t), \varphi)=\widetilde{\mathcal{F}}(t ; \varphi) \quad \forall \varphi \in V_{0}^{(t)},
$$

with

$$
a_{\boldsymbol{\sigma}, \boldsymbol{\beta}, \kappa}(t ; w, \varphi):=\int_{\Omega} \nabla \varphi \cdot(\boldsymbol{\sigma} \nabla w) \mathrm{d} \Omega+\int_{\Omega} \varphi \boldsymbol{\beta} \cdot \nabla w \mathrm{~d} \Omega+\int_{\Omega} \varphi \kappa w \mathrm{~d} \Omega
$$

3 A more general result is provided in [29] considering a weaker condition on the dilatation of the Dirichlet boundary and solutions in Besov spaces $[2,8,20]$. 
and

$$
\widetilde{\mathcal{F}}(t ; \varphi):=\int_{\Omega} \varphi f(t) \mathrm{d} \Omega+\int_{\Gamma_{N}^{(t)}} \varphi G(t) \mathrm{d} \Gamma-a_{\boldsymbol{\sigma}, \boldsymbol{\beta}, \kappa}(t ; \bar{g}(t), \varphi) .
$$

At this point, we introduce a time discretization of equation (2.10), by using the BE scheme ${ }^{4}$, yielding the semi-discrete problem. Let $N_{T} \in \mathbb{N}$ be a given integer and consider an uniform partition of the time interval $(0, T)$ into subintervals $\left\{\left[t_{n}, t_{n+1}\right)\right\}_{n=0}^{N_{T}-1}$ of size $\Delta t:=T / N_{T}$, with $t_{n}:=n \Delta t$, for $n=0, \ldots, N_{T}$. By evaluating the data $f, g$, and $G$ at the time $t_{n}$ as $f^{n}, g^{n}$, and $G^{n}$, for $n=1, \ldots, N_{T}$, and by setting $u^{0}=u_{0}$ in $\Omega$, we solve recursively the following family of elliptic problems in the unknowns $u^{n} \in V^{\left(t^{n}\right)}$, for $n=1, \ldots, N_{T}$, reading:

$$
\text { find } w^{n} \in V_{0}^{\left(t^{n}\right)}: \quad \frac{1}{\Delta t}\left(\left(w^{n}-w^{n-1}\right), \varphi\right)_{\mathrm{L}^{2}(\Omega)}+a_{\boldsymbol{\sigma}, \boldsymbol{\beta}, \kappa}\left(t^{n} ; w^{n}, \varphi\right)=\widetilde{\mathcal{F}}\left(t^{n} ; \varphi\right) \quad \forall \varphi \in V_{0}^{\left(t^{n}\right)} ;
$$

with $u^{n}=w^{n}+\bar{g}^{n}$.

Finally, in order to obtain the full discrete FE formulation, we consider a regular family of quasi-uniform triangulations $\left\{\mathcal{T}_{h}^{n}\right\}_{h}$ of $\Omega$, denoting with $h_{T}:=\operatorname{diam} T$ the diameter of any mesh element $T \in \mathcal{T}_{h}^{n}$ and by $h^{n}:=\max _{T \in \mathcal{T}_{h}^{n}} h_{T}$ the mesh size at time $t_{n}([26,33])$. Moreover, we assume that for any mesh size $h$, the mesh $\mathcal{T}_{h}^{n}$ is conforming with the boundary $\Gamma \equiv \partial \Omega$ and we denote by $\mathcal{E}_{h}^{n}$ the corresponding triangulation on the interface, whose elements (edges or faces) are denoted by $\Gamma_{b}$ for $b=1, \ldots, N_{b}^{h}$ with associated diameter $h_{b}^{n}:=\operatorname{diam} \Gamma_{b}^{n}$, respectively. We remark that the mesh $\mathcal{T}_{h}^{n}$ varies or changes with the time $t^{n}$, as well as $\mathcal{E}_{h}^{n}$. Therefore, for any $n=1, \ldots, N_{T}$, the full discrete problem (2.6) is defined by looking for a $\mathrm{FE}$ approximated solution $u_{h}^{n}$ of equation (2.13) in the FE space of degree $k \geq 1$ defined as:

$$
\mathcal{V}_{0, h}^{n}:=\mathcal{X}_{h}^{n} \cap V_{0}^{\left(t^{n}\right)},
$$

where $\mathcal{X}_{h}^{n}:=\left\{\varphi_{h} \in \mathrm{C}^{0}(\bar{\Omega}):\left.\varphi_{h}\right|_{T} \in \mathbb{P}_{k} \quad \forall T \in \mathcal{T}_{h}^{n}\right\}$ and $\mathbb{P}_{k}$ denotes the space of polynomials of degree less than or equal to $k$. We remark that the FE space $\mathcal{X}_{h}^{n}$ depends on the mesh $\mathcal{T}_{h}^{n}$, which may change at each time step in order to match $\Gamma_{D}^{\left(t^{n}\right)}$. Then, the full discrete problem reads, for $n=1, \ldots, N_{T}$ :

$$
\text { find } u_{h}^{n} \in V^{\left(t^{n}\right)}: \quad\left(w_{h}^{n}, \varphi_{h}\right)+\Delta t a_{\boldsymbol{\sigma}, \boldsymbol{\beta}, \kappa}\left(t^{n} ; w_{h}^{n}, \varphi_{h}\right)=\Delta t \widetilde{\mathcal{F}}\left(t^{n} ; \varphi_{h}\right)+\left(w_{h}^{n-1}, \varphi_{h}\right) \quad \forall \varphi_{h} \in \mathcal{V}_{0, h}^{n} ;
$$

with $u_{h}^{n}=w_{h}^{n}+\bar{g}_{h}^{n}$, given $u_{h}^{0} \in \mathcal{V}_{h}$, where $\widetilde{\mathcal{F}}$ involves the approximate lifting function $\bar{g}^{n} \in \mathcal{V}_{g, h}^{n}:=\mathcal{X}_{h} \cap V_{g}^{\left(t^{n}\right)}$, e.g. obtained by a $\mathrm{L}^{2}$ projection technique.

Remark 2.2. The FE spaces of equation (2.14) are time varying function spaces. Specifically, depending on the measure of $\Gamma_{D}^{\left(t_{n}\right)}$, the number of degrees of freedom associated to the FE space $\mathcal{V}_{0, h}^{(n)}$ changes accordingly, as well as the size and the structure of the associated discrete problem.

Remark 2.3. When implementing problem (2.15) by using the standard Galerkin FE formulation with time varying function spaces, the FE space can only be defined after the Dirichlet boundary $\Gamma_{D}^{\left(t^{n}\right)}$ is prescribed at time $t^{n}$. Therefore, one needs to re-mesh and re-define a new FE space for any discrete time $t^{n}$, for all $n=1, \ldots, N_{T}$. Moreover, for any $t^{n}$, suitable injection and projection operators need to be introduced to account for the variation of the mesh $\mathcal{T}_{h}^{n}$. In practice, to account for this variation, we need to introduce a mapping $T^{n}$ acting on the data $u^{n-1}$ as $T^{n}: \mathcal{V}_{g, h}^{n-1} \stackrel{I_{h}^{n}}{\rightarrow} \mathrm{H}^{1}(\Omega) \stackrel{P_{h}^{n}}{\longrightarrow} \mathcal{V}_{g, h}^{n}$, where $I_{h}^{n}$ denotes the injection operator of the solution $u^{n-1} \in \mathcal{V}_{g, h}^{n-1}$ into $\mathrm{H}^{1}(\Omega)$, while $P_{h}^{n}$ is the projection operator from $\mathrm{H}^{1}(\Omega)$ onto the FE space $\mathcal{V}_{g, h}^{n}$. Accounting for such operators in commercial software or FE codes is neither straightforward nor computationally efficient.

\footnotetext{
${ }^{4}$ We use the BE scheme in the framework of the proof of Theorem 2.1 in [29], for which the author shows that, under suitable hypotheses, the continuous and piecewise linear (with respect to time) solution of the semi-discrete problem converges to the solution $u(t)$ of equation (2.6) when the time step tends to zero.
} 


\section{Nitsche's Method FOR MIXED TIME VARYing BCs}

In this section, we propose the Nitsche's method for the treatment of the mixed time varying BCs of equation (2.6). Following what done in [17] for elliptic PDEs, we consider equation (2.6a) endowed with a generalized Robin BCs in the form:

$$
-\boldsymbol{\Phi}_{\mathrm{diff}}(u(\mathbf{x}, t)) \cdot \mathbf{n}+\gamma(\mathbf{x}, t) u(\mathbf{x}, t)=G(\mathbf{x}, t)+\gamma(\mathbf{x}, t) g(\mathbf{x}, t) \quad \text { on } \Sigma,
$$

where $\gamma: \Sigma \rightarrow(0,+\infty) \subseteq \mathbb{R}$. Indeed, given $f \in \mathrm{L}^{2}\left(0, T ; \mathrm{L}^{2}(\Omega)\right), u_{0} \in \mathrm{H}^{1}(\Omega), g \in \mathrm{L}^{2}\left(0, T ; \mathrm{H}^{1 / 2}\left(\Gamma_{D}^{(t)}\right)\right)$, and $G \in \mathrm{L}^{2}\left(0, T ; \mathrm{L}^{2}\left(\Gamma_{N}^{(t)}\right)\right)$, we consider the problem:

find $u: \Omega \times(0, T) \rightarrow \mathbb{R}:$

$$
\begin{cases}\frac{\partial u}{\partial t}(\mathbf{x}, t)+\mathcal{L} u(\mathbf{x}, t)=f(\mathbf{x}, t) & \text { in } \Omega \times(0, T), \\ -\boldsymbol{\Phi}_{\mathrm{diff}}(u(\mathbf{x}, t)) \cdot \mathbf{n}+\gamma(\mathbf{x}, t) u(\mathbf{x}, t)=G(\mathbf{x}, t)+\gamma(\mathbf{x}, t) g(\mathbf{x}, t) & \text { on } \Sigma, \\ u(0)=u_{0} & \text { in } \Omega,\end{cases}
$$

with $u_{0}(\mathbf{x})=g(\mathbf{x}, 0)$ on $\Gamma_{D}^{(0)}$. We notice that in the limit $\gamma \rightarrow 0$, equation (3.2b) tends to the pure Neumann BC of equation (2.6c), while, in the limit $\gamma \rightarrow \infty$, we recover the pure Dirichlet BC of equation (2.6b). Problem (3.2) can therefore be regarded as more general than (2.6).

\subsection{The Nitsche's method: Spatial discretization}

For the spatial discretization of equation (3.2), we introduce a FE approximation based on the Nitsche's method $[17,22]$ to treat the time varying BCs by looking for a solution of the weak counterpart of equation (3.2) in $\mathrm{H}^{1}(\Omega)$ with function spaces independent of time. We introduce the FE space of degree $k \geq 1$ defined as:

$$
\mathcal{V}_{h}:=\mathcal{X}_{h} \cap \mathrm{H}^{1}(\Omega),
$$

with $\mathcal{X}_{h}$ being the FE space of Lagrangian basis functions defined over the mesh $\mathcal{T}_{h}$, which is now fixed in $\Omega$ and in time; $h:=\max _{T \in \mathcal{T}_{h}} h_{T}$ represents the mesh size, $h_{T}:=\operatorname{diam} T$ the diameter of any element $T \in \mathcal{T}_{h}, \mathcal{E}_{h}$ the triangulation on the boundary, whose elements (edges or faces) are denoted by $\Gamma_{b}$ for $b=1, \ldots, N_{b}^{h}$ with associated diameter $h_{b}:=\operatorname{diam} \Gamma_{b}$. Moreover, let $T_{b}$, for $b=1, \ldots, N_{b}^{h}$ be the boundary triangle of $\mathcal{T}_{h}$ associated to $\Gamma_{b}$, i.e. $\Gamma_{b}:=T_{b} \cap \Gamma$.

\subsubsection{The semi-discrete problem}

We consider the following problem, for all $t \in(0, T)$ :

$$
\text { find } u_{h}(t) \in \mathcal{V}_{h}: \quad\left(\frac{\partial u_{h}}{\partial t}(t), \varphi_{h}\right)+a_{h}\left(t ; u_{h}(t), \varphi_{h}\right)=\mathcal{F}_{h}\left(t ; \varphi_{h}\right) \quad \forall \varphi_{h} \in \mathcal{V}_{h},
$$

where:

$$
\begin{aligned}
a_{h}\left(t ; u_{h}(t), \varphi_{h}\right):= & a_{\boldsymbol{\sigma}, \boldsymbol{\beta}, \kappa}\left(t ; u_{h}(t), \varphi_{h}\right)+\sum_{b=1}^{N_{b}^{h}}\left[\int_{\Gamma_{b}} \varphi_{h}\left(-\frac{\gamma h_{b}}{\xi+\gamma h_{b}}\right) \boldsymbol{\sigma} \nabla u_{h} \cdot \mathbf{n} \mathrm{d} \Gamma\right. \\
& +\int_{\Gamma_{b}}\left(\boldsymbol{\Phi}_{i n}^{*}\left(\varphi_{h}\right) \cdot \mathbf{n}\right)\left(-\frac{\gamma h_{b}}{\xi+\gamma h_{b}}\right) u_{h} \mathrm{~d} \Gamma+\int_{\Gamma_{b}} \varphi_{h}\left(\frac{|\boldsymbol{\sigma}| \xi \gamma}{\xi+\gamma h_{b}}\right) u_{h} \mathrm{~d} \Gamma \\
& \left.+\int_{\Gamma_{b}}\left(\boldsymbol{\Phi}_{i n}^{*}\left(\varphi_{h}\right) \cdot \mathbf{n}\right)\left(-\frac{h_{b}}{|\boldsymbol{\sigma}|\left(\xi+\gamma h_{b}\right)}\right) \boldsymbol{\sigma} \nabla u_{h} \cdot \mathbf{n} \mathrm{d} \Gamma\right]
\end{aligned}
$$


and:

$$
\begin{aligned}
\mathcal{F}_{h}\left(t ; \varphi_{h}\right):= & \mathcal{F}\left(t ; \varphi_{h}\right)+\sum_{b=1}^{N_{b}^{h}}\left[\int_{\Gamma_{b}}\left(\boldsymbol{\Phi}_{i n}^{*}\left(\varphi_{h}\right) \cdot \mathbf{n}\right)\left(-\frac{\gamma h_{b}}{\xi+\gamma h_{b}}\right) g \mathrm{~d} \Gamma+\int_{\Gamma_{b}} \varphi_{h}\left(\frac{|\boldsymbol{\sigma}| \xi \gamma}{\xi+\gamma h_{b}}\right) g \mathrm{~d} \Gamma\right. \\
& \left.+\int_{\Gamma_{b}}\left(\boldsymbol{\Phi}_{i n}^{*}\left(\varphi_{h}\right) \cdot \mathbf{n}\right)\left(-\frac{h_{b}}{|\boldsymbol{\sigma}|\left(\xi+\gamma h_{b}\right)}\right) G \mathrm{~d} \Gamma+\int_{\Gamma_{b}} \varphi_{h}\left(\frac{\xi}{\xi+\gamma h_{b}}\right) G \mathrm{~d} \Gamma\right],
\end{aligned}
$$

with $a_{\boldsymbol{\sigma}, \boldsymbol{\beta}, \kappa}\left(t ; u_{h}, \varphi_{h}\right)$ defined in equation $(2.11), \mathcal{F}\left(t ; \varphi_{h}\right):=\int_{\Omega} \varphi_{h} f \mathrm{~d} \Omega, \chi_{i n}$ and $\chi_{\text {out }}$ the characteristic functions of the subsets of the boundary corresponding to the inflow and outflow parts, respectively:

$$
\chi_{i n}: \Sigma \rightarrow\{0,1\}, \quad \chi_{i n}(\mathbf{x}, t):=\left\{\begin{array}{ll}
1 & \text { if } \boldsymbol{\beta}(\mathbf{x}, t) \cdot \mathbf{n}<0, \\
0 & \text { otherwise },
\end{array} \quad \text { and } \quad \chi_{\text {out }}:=1-\chi_{\text {in }},\right.
$$

and $\boldsymbol{\Phi}_{i n}^{*}$ is the adjoint of the inflow flux tensor:

$$
\boldsymbol{\Phi}_{i n}^{*}(u(\mathbf{x}, t)):=\boldsymbol{\sigma}(\mathbf{x}, t) \nabla u(\mathbf{x}, t)+u(\mathbf{x}, t) \boldsymbol{\beta}(\mathbf{x}, t) \chi_{i n}(\mathbf{x}, t) ;
$$

moreover, $\xi: \bigcup_{b=1}^{N_{b}^{h}} \Gamma_{b} \times(0, T) \rightarrow(0,+\infty)$ is the penalty function.

Remark 3.1. The first boundary term of equation (3.5) is a consistency term, while the remaining terms of equations (3.5) and (3.6) ensure a weak enforcement of the BCs. The case of pure Neumann BC, i.e. when $\Sigma_{N} \equiv \Sigma$, is recovered for $\gamma(\mathbf{x}, t) \rightarrow 0^{+}$a.e. $(\mathbf{x}, t) \in \Sigma$. Indeed, in that case, equation (3.4) becomes:

$$
\text { find } \begin{aligned}
u_{h}(t) \in \mathcal{V}_{h}: & \left(\frac{\partial u_{h}}{\partial t}(t), \varphi_{h}\right)+a_{\boldsymbol{\sigma}, \boldsymbol{\beta}, \kappa}\left(t ; u_{h}, \varphi_{h}\right)+\sum_{b=1}^{N_{b}^{h}} \int_{\Gamma_{b}}\left(\boldsymbol{\Phi}_{i n}^{*}\left(\varphi_{h}\right) \cdot \mathbf{n}\right)\left(-\frac{h_{b}}{|\boldsymbol{\sigma}| \xi}\right) \boldsymbol{\sigma} \nabla u_{h} \cdot \mathbf{n} \mathrm{d} \Gamma \\
& =\mathcal{F}\left(t ; \varphi_{h}\right)+\int_{\Gamma} \varphi_{h} G \mathrm{~d} \Gamma+\sum_{b=1}^{N_{b}^{h}} \int_{\Gamma_{b}}\left(\boldsymbol{\Phi}_{i n}^{*}\left(\varphi_{h}\right) \cdot \mathbf{n}\right)\left(-\frac{h_{b}}{|\boldsymbol{\sigma}| \xi}\right) G \mathrm{~d} \Gamma \quad \forall \varphi_{h} \in \mathcal{V}_{h} .
\end{aligned}
$$

We notice that the last terms on either side of equation (3.9) represent additional terms with respect to the standard formulation, which however do not affect the consistency of the method. The case of a pure Dirichlet $\mathrm{BC}$, i.e. when $\Sigma_{D} \equiv \Sigma$, is recovered for $\gamma(\mathbf{x}, t) \rightarrow+\infty$ a.e. $(\mathbf{x}, t) \in \Sigma$. In this case, equation (3.4) reads:

$$
\text { find } \begin{aligned}
u_{h}(t) \in \mathcal{V}_{h}: & \left(\frac{\partial u_{h}}{\partial t}(t), \varphi_{h}\right)+a_{\boldsymbol{\sigma}, \boldsymbol{\beta}, \kappa}\left(t ; u_{h}, \varphi_{h}\right)+\sum_{b=1}^{N_{b}^{h}} \int_{\Gamma_{b}}-\varphi_{h}\left(\boldsymbol{\sigma} \nabla u_{h} \cdot \mathbf{n}\right) \mathrm{d} \Gamma \\
& +\sum_{b=1}^{N_{b}^{h}} \int_{\Gamma_{b}}-\left(\boldsymbol{\Phi}_{i n}^{*}\left(\varphi_{h}\right) \cdot \mathbf{n}\right) u_{h} \mathrm{~d} \Gamma+\sum_{b=1}^{N_{b}^{h}} \int_{\Gamma_{b}} \varphi_{h}\left(\frac{|\boldsymbol{\sigma}| \xi}{h_{b}}\right) u_{h} \mathrm{~d} \Gamma \\
& =\mathcal{F}\left(t ; \varphi_{h}\right)+\sum_{b=1}^{N_{b}^{h}} \int_{\Gamma_{b}}-\left(\boldsymbol{\Phi}_{i n}^{*}\left(\varphi_{h}\right) \cdot \mathbf{n}\right) g \mathrm{~d} \Gamma+\sum_{b=1}^{N_{b}^{h}} \int_{\Gamma_{b}} \varphi_{h}\left(\frac{|\boldsymbol{\sigma}| \xi}{h_{b}}\right) g \mathrm{~d} \Gamma .
\end{aligned}
$$

Equation (3.10) corresponds to the weak treatment of the Dirichlet BCs as considered e.g. in [6]. As a matter of fact, the weak imposition of Dirichlet BCs of [6] represents a particular case of the more general scheme proposed in this paper. Weak imposition of Dirichlet BCs is easily accommodated in the context of the Discontinuous Galerkin (DG) method [25,27], where the inter-element continuity of the solution is weakly enforced. In particular, in view of the analysis of the penalty terms added to the variational formulation, we refer the interested reader to $[3,27]$. 
Remark 3.2. Concerning the penalty function $\xi=\xi(\mathbf{x}, t)$, we attribute a different role to $\xi$ on the boundary of the space-time cylinder $\Sigma$ where we impose either Dirichlet or Neumann BCs. Indeed, from definitions (3.5) and (3.6), we infer that, for the weak imposition of the Dirichlet $\mathrm{BC}$ on $\Gamma$, the bigger the value of $\xi$, the more significant is the penalization on the Dirichlet data $g$. Conversely, for the Neumann $\mathrm{BC}$ on $\Gamma$, the larger the value of $\xi$, the smaller is the contribution of the additional consistency terms, see equation (3.9).

We remark that equation (3.4) considers a different treatment of the inflow boundary part with respect to the outflow one, with the aim of controlling both the regimes of dominating advection or diffusion. The scaling with respect to $|\boldsymbol{\sigma}|$ of the boundary terms of equations (3.5) and (3.6) allows to consider the correct dimensions in the formulation. The weak treatment of the Dirichlet BCs at the inflow and outflow boundaries, which is obtained for $\gamma(\mathbf{x}, t) \rightarrow+\infty$, can be justified analogously to e.g. [6]. For the analysis of the Nitsche's method, we impose the following restrictions on $\xi: \bigcup_{b=1}^{N_{b}^{h}} \Gamma_{b} \times(0, T) \rightarrow(0,+\infty) ; \xi$ is a measurable function for which there exists two positive constants $\bar{\xi}_{0}$ and $\bar{\xi}_{\infty}$ such that:

$$
0<\bar{\xi}_{0} \leq \xi(\mathbf{x}, t) \leq \bar{\xi}_{\infty}<+\infty, \quad \forall(\mathbf{x}, t) \in \bigcup_{b=1}^{N_{b}^{h}} \Gamma_{b} \times(0, T) .
$$

Then, let us define the following weighting functions for the Nitsche's method:

$$
\xi_{k}^{b}: \Gamma \times(0, T) \rightarrow \mathbb{R}:(\mathbf{x}, t) \mapsto \frac{\delta_{k}^{b}}{\xi(\mathbf{x}, t)+\gamma(\mathbf{x}, t) h_{b} \chi_{\Gamma_{b}}(\mathbf{x})},
$$

for $k=1, \ldots, 4$, with:

$$
\delta_{1}^{b}:=\gamma(\mathbf{x}, t) h_{b} \chi_{\Gamma_{b}}(\mathbf{x}), \delta_{2}^{b}:=\xi(\mathbf{x}, t) \gamma(\mathbf{x}, t), \delta_{3}^{b}:=h_{b} \chi_{\Gamma_{b}}(\mathbf{x}), \quad \text { and } \quad \delta_{4}^{b}:=\xi(\mathbf{x}, t),
$$

for all $b=1, \ldots, N_{b}^{h}$ and with $\chi_{\Gamma_{b}}$ the characteristic functions of the subset $\Gamma_{b}$, i.e. $\chi_{\Gamma_{b}}: \Gamma \rightarrow\{0,1\}$ : $\mathbf{x} \mapsto\left\{\begin{array}{ll}1 & \text { if } \mathbf{x} \in \Gamma_{b}, \\ 0 & \text { otherwise }\end{array}\right.$. For the weighting functions (3.12), we have the following upper bounds:

$$
\begin{array}{lll}
\left|\xi_{1}^{b}(\mathbf{x}, t)\right| \leq 2, & \left|\xi_{2}^{b}(\mathbf{x}, t)\right| \leq 2 \frac{\bar{\xi}_{\infty}}{\min _{b} h_{b}} \lesssim \frac{\bar{\xi}_{\infty}}{h}, & \\
\left|\xi_{3}^{b}(\mathbf{x}, t)\right| \leq \frac{\max _{b} h_{b}}{\bar{\xi}_{0}} \lesssim \frac{h}{\bar{\xi}_{0}}, & \left|\xi_{4}^{b}(\mathbf{x}, t)\right| \leq 1, & \forall(\mathbf{x}, t) \in \Gamma \times(0, T),
\end{array}
$$

where equation (3.14) for $\xi_{2}^{b}$ and $\xi_{3}^{b}$ follow from the quasi-uniformity of the family of triangulations $\mathcal{T}_{h}$.

\subsubsection{Some preliminary lemmas}

We recall the following inverse inequalities for traces of finite element functions from [3,33]. Let $\widetilde{\Gamma} \subseteq \partial \Omega$, then there exist positive constants $\widetilde{C}_{1}$ and $\widetilde{C}_{2}>0$ independent of $h$, but possibly dependent on the FE degree $k$ such that:

$$
\|\phi\|_{\mathrm{L}^{2}(\widetilde{\Gamma})}^{2} \leq \widetilde{C}_{1} h^{-1}\|\phi\|_{\mathrm{L}^{2}(\Omega)}^{2} \quad \text { and } \quad\|\nabla \phi \cdot \mathbf{n}\|_{\mathrm{L}^{2}(\widetilde{\Gamma})}^{2} \leq \widetilde{C}_{2} h^{-1}|\phi|_{\mathrm{H}^{1}(\Omega)}^{2} \quad \forall \phi \in \mathcal{V}_{h} .
$$

Moreover, for $b=1, \ldots, N_{b}^{h}$, there exist constants $\bar{C}_{1}$ and $\bar{C}_{2}>0$ dependent on $k$, but independent of $h_{b}$, such that:

$$
\begin{aligned}
\|\phi\|_{\mathrm{L}^{2}\left(\Gamma_{b}\right)}^{2} & \leq \bar{C}_{1} h_{b}^{-1}\|\phi\|_{\mathrm{L}^{2}\left(T_{b}\right)}^{2} & & \forall \phi \in \mathcal{V}_{h}, \\
\|\nabla \phi \cdot \mathbf{n}\|_{\mathrm{L}^{2}\left(\Gamma_{b}\right)}^{2} & \leq \bar{C}_{2} h_{b}^{-1}|\phi|_{\mathrm{H}^{1}\left(T_{b}\right)}^{2} & & \forall \phi \in \mathcal{V}_{h}
\end{aligned}
$$


Finally, thanks to the quasi-uniformity of $\mathcal{T}_{h}$, the following inverse inequality holds [33] for a positive constant $\bar{C}$ independent of $h$ :

$$
|\phi|_{\mathrm{H}^{1}(\Omega)}^{2} \leq \bar{C} h^{-2}\|\phi\|_{\mathrm{L}^{2}(\Omega)}^{2} \quad \forall \phi \in \mathcal{V}_{h}
$$

For our analysis, we introduce the following $h$-dependent norm:

$$
\|v\|_{h}:=\left(|v|_{\mathrm{H}^{1}(\Omega)}^{2}+\sum_{b=1}^{N_{b}^{h}}\|\zeta v\|_{\mathrm{L}^{2}\left(\Gamma_{b}\right)}^{2}\right)^{1 / 2}
$$

where, for the sake of simplicity, we have introduced from equation (3.12), with $k=2$, the function:

$$
\zeta: \Sigma \rightarrow \mathbb{R}:(\mathbf{x}, t) \mapsto \sqrt{\xi_{2}^{b}(\mathbf{x}, t)} .
$$

We recall the following result, consequence of the Peetre-Tartar Lemma [15], from which we deduce the successive lemmas.

Lemma 3.3. Let $\mathcal{O}$ be a general open bounded connected set with Lipschitz boundary and let $F$ be a linear functional on $\mathrm{H}^{1}(\mathcal{O})$ whose restriction on constant functions is not null; then, there exists a positive constant $C_{\mathcal{O}}>0$, dependent on the domain $\mathcal{O}$, such that:

$$
C_{\mathcal{O}}\|\varphi\|_{\mathrm{H}^{1}(\mathcal{O})} \leq|\varphi|_{\mathrm{H}^{1}(\mathcal{O})}+|F(\varphi)| \quad \forall \varphi \in \mathrm{H}^{1}(\mathcal{O}) .
$$

Lemma 3.4. Under the hypotheses of Lemma 3.3, for $\mathcal{O}=T_{b}$, there exists a constant $C_{\Omega_{B}}>0$, related to the boundary elements of the mesh $T_{b}$ for $b=1, \ldots, N_{b}^{h}$, such that for the norm $\|\cdot\|_{h}$ of equation (3.18):

$$
\sum_{b=1}^{N_{b}^{h}}\left\|\varphi_{h}\right\|_{\mathrm{H}^{1}\left(T_{b}\right)}^{2} \leq C_{\Omega_{B}}\left\|\varphi_{h}\right\|_{h}^{2} \quad \forall \varphi_{h} \in \mathcal{V}_{h} .
$$

Proof. By applying Lemma 3.3 for $\mathcal{O} \equiv T_{b}$ and $F(\varphi)=\frac{1}{h_{b}} \int_{\Gamma_{b}} \zeta \varphi \mathrm{d} \Gamma$, we have:

$$
|F(\varphi)|=\frac{1}{h_{b}}\left|\int_{\Gamma_{b}} \zeta \varphi \mathrm{d} \Gamma\right| \leq \frac{1}{h_{b}}\|1\|_{\mathrm{L}^{2}\left(\Gamma_{b}\right)}\|\zeta \varphi\|_{\mathrm{L}^{2}\left(\Gamma_{b}\right)}=\|\zeta \varphi\|_{\mathrm{L}^{2}\left(\Gamma_{b}\right)},
$$

and, for any $b=1, \ldots, N_{b}^{h}$, there exists $C_{T_{b}}>0$, such that:

$$
C_{T_{b}}\left\|\varphi_{h}\right\|_{\mathrm{H}^{1}\left(T_{b}\right)} \leq\left|\varphi_{h}\right|_{\mathrm{H}^{1}\left(T_{b}\right)}+\left|F\left(\varphi_{h}\right)\right| \leq\left|\varphi_{h}\right|_{\mathrm{H}^{1}\left(T_{b}\right)}+\left\|\zeta \varphi_{h}\right\|_{\mathrm{L}^{2}\left(\Gamma_{b}\right)} ;
$$

therefore, $\frac{C_{T_{b}}^{2}}{2}\left\|\varphi_{h}\right\|_{\mathrm{H}^{1}\left(T_{b}\right)}^{2} \leq\left|\varphi_{h}\right|_{\mathrm{H}^{1}\left(T_{b}\right)}^{2}+\left\|\zeta \varphi_{h}\right\|_{\mathrm{L}^{2}\left(\Gamma_{b}\right)}^{2}$. By summing the latter over $b=1, \ldots, N_{b}^{h}$, we have:

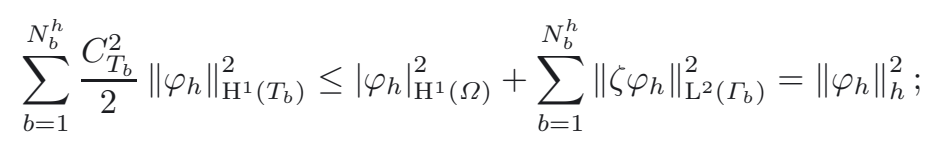

by setting $C_{\Omega_{B}}:=\frac{2}{\min _{b} C_{T_{b}}^{2}}$, equation (3.21) follows.

Lemma 3.5. Under the hypotheses of Lemma 3.3, for $\mathcal{O}=\Omega$, there exists a constant $C_{\Omega}>0$ such that, for the norm $\|\cdot\|_{h}$ of equation (3.18):

$$
\begin{array}{rlrl}
\left\|\varphi_{h}\right\|_{\mathrm{H}^{1}(\Omega)}^{2} & \leq \frac{2}{C_{\Omega}^{2}}\left\|\varphi_{h}\right\|_{h}^{2} & \forall \varphi_{h} \in \mathcal{V}_{h}, \\
\left\|\varphi_{h}\right\|_{\mathrm{L}^{2}(\Gamma)}^{2} \leq \frac{2 C_{\mathrm{tr}}^{2}}{C_{\Omega}^{2}}\left\|\varphi_{h}\right\|_{h}^{2} & \forall \varphi_{h} \in \mathcal{V}_{h},
\end{array}
$$

with $C_{\mathrm{tr}}$ trace constant, such that:

$$
\left\|\varphi_{h}\right\|_{\mathrm{L}^{2}(\Gamma)} \leq C_{\mathrm{tr}}\left\|\varphi_{h}\right\|_{\mathrm{H}^{1}(\Omega)} .
$$


Proof. By applying Lemma 3.3 for $\mathcal{O} \equiv \Omega$ and $F(\varphi):=\frac{1}{\sqrt{2 N_{b}^{h}} \max _{b} h_{b}} \sum_{b=1}^{N_{b}^{h}} \int_{\Gamma_{b}} \zeta \varphi \mathrm{d} \Gamma$, we obtain:

$$
C_{\Omega}\left\|\varphi_{h}\right\|_{\mathrm{H}^{1}(\Omega)} \leq\left|\varphi_{h}\right|_{\mathrm{H}^{1}(\Omega)}+\left|F\left(\varphi_{h}\right)\right| \leq\left|\varphi_{h}\right|_{\mathrm{H}^{1}(\Omega)}+\frac{1}{\sqrt{2 N_{b}^{h}}} \sum_{b=1}^{N_{b}^{h}}\left\|\zeta \varphi_{h}\right\|_{\mathrm{L}^{2}\left(\Gamma_{b}\right)},
$$

where the upper bound for $\left|F\left(\varphi_{h}\right)\right|$ follows by the triangle inequality and Schwarz inequality $|F(\varphi)| \leq \frac{1}{\sqrt{2 N_{b}^{h}}} \sum_{b=1}^{N_{b}^{h}}\|\zeta \varphi\|_{L^{2}\left(\Gamma_{b}\right)}$. Furthermore, since $\left(\sum_{b=1}^{N_{b}^{h}}\|\zeta \varphi\|_{L^{2}\left(\Gamma_{b}\right)}\right)^{2} \leq 2 N_{b}^{h} \sum_{b=1}^{N_{b}^{h}}\|\zeta \varphi\|_{L^{2}\left(\Gamma_{b}\right)}^{2}$, we obtain:

$$
C_{\Omega}^{2}\left\|\varphi_{h}\right\|_{\mathrm{H}^{1}(\Omega)}^{2} \leq 2\left|\varphi_{h}\right|_{\mathrm{H}^{1}(\Omega)}^{2}+2 \sum_{b=1}^{N_{b}^{h}}\left\|\zeta \varphi_{h}\right\|_{\mathrm{L}^{2}\left(\Gamma_{b}\right)}^{2}=2\left\|\varphi_{h}\right\|_{h}^{2},
$$

yielding (3.25a); (3.25b) follows from (3.25a) and (3.26).

Lemma 3.6. There exist positive constants $C_{1}^{(1)}$ and $C_{1, h}^{(2)}>0$ such that:

$$
C_{1}^{(1)}\left\|\varphi_{h}\right\|_{\mathrm{H}^{1}(\Omega)} \leq\left\|\varphi_{h}\right\|_{h} \leq C_{1, h}^{(2)}\left\|\varphi_{h}\right\|_{\mathrm{H}^{1}(\Omega)} \quad \forall \varphi_{h} \in \mathcal{V}_{h}
$$

where $C_{1}^{(1)}$ is independent of $h$, while $C_{1, h}^{(2)}:=\sqrt{1+\frac{2 C_{\mathrm{tr}}^{2} \bar{\xi}_{\infty}}{\min _{b} h_{b}}}$ scales as $h^{-1 / 2}$ for $h \rightarrow 0$.

Proof. By using Lemma 3.5, we obtain the left inequality of equation (3.29), with $C_{1}^{(1)}:=\frac{C_{\Omega}}{\sqrt{2}}$. Using the bound (3.14) for the function $\xi_{2}^{b}$ and the trace inequality (3.26), we obtain:

$$
\sum_{b=1}^{N_{b}^{h}}\|\zeta \varphi\|_{\mathrm{L}^{2}\left(\Gamma_{b}\right)}^{2} \leq \frac{2 \bar{\xi}_{\infty}}{\min _{b} h_{b}} \sum_{b=1}^{N_{b}^{h}}\|\varphi\|_{\mathrm{L}^{2}\left(\Gamma_{b}\right)}^{2} \leq \frac{2 \bar{\xi}_{\infty}}{\min _{b} h_{b}}\|\varphi\|_{\mathrm{L}^{2}(\Gamma)}^{2} \leq \frac{2 C_{\mathrm{tr}}^{2} \bar{\xi}_{\infty}}{\min _{b} h_{b}}\|\varphi\|_{\mathrm{H}^{1}(\Omega)}^{2} .
$$

Lemma 3.7. For the norm $\|\cdot\|_{h}$, there exists a constant $\widetilde{C}_{0}>0$ independent of $h$ such that:

$$
\left\|\varphi_{h}\right\|_{h} \leq \widetilde{C}_{0} h^{-1}\left\|\varphi_{h}\right\|_{L^{2}(\Omega)} \quad \forall \varphi_{h} \in \mathcal{V}_{h} .
$$

Proof. By considering the inverse inequalities (3.16a) and (3.17) together with the second upper bound of the inequality (3.30), we have:

$$
\left\|\varphi_{h}\right\|_{h}^{2} \leq\left|\varphi_{h}\right|_{\mathrm{H}^{1}(\Omega)}^{2}+\frac{2 \bar{\xi}_{\infty}}{\min _{b} h_{b}}\|\varphi\|_{\mathrm{L}^{2}(\Gamma)}^{2} \leq \bar{C} h^{-2}\left\|\varphi_{h}\right\|_{\mathrm{L}^{2}(\Omega)}^{2}+2 \bar{\xi}_{\infty} \widetilde{C}_{1} h^{-2}\left\|\varphi_{h}\right\|_{\mathrm{L}^{2}(\Omega)}^{2} .
$$

The latter inequality follows from the hypothesis of the quasi-uniformity of $\left\{\mathcal{T}_{h}\right\}_{h}$; indeed, there exists a constant $\tau>0$ such that $\min _{T \in \mathcal{T}_{h}} h_{T} \geq \tau h$ for $h:=\max _{T \in \mathcal{T}_{h}} h_{T}$ for which we embed the constant $\tau$ in $\widetilde{C}_{1}$. The thesis follows with $\widetilde{C}_{0}^{2}:=\max \left\{\bar{C}, 2 \bar{\xi}_{\infty} \widetilde{C}_{1}\right\}$.

\subsubsection{Analysis of the Nitsche's method: consistency and well posedness}

We start by verifying the consistency of the formulation (3.4) with respect to problem (2.6).

Lemma 3.8. Let $u_{h}(t) \in \mathcal{V}_{h}$ be solution of (3.4) and $u(t): \Omega \times(0, T) \rightarrow \mathbb{R}$ the solution of (3.2), then, for all $t \in(0, T)$, it holds:

$$
\left(\frac{\partial}{\partial t}\left(u_{h}(t)-u(t)\right), \varphi_{h}\right)+a_{h}\left(t ;\left(u_{h}(t)-u(t)\right), \varphi_{h}\right)=0 \quad \forall \varphi_{h} \in \mathcal{V}_{h},
$$

where $a_{h}(t ; \cdot, \cdot)$ is defined in equation (3.5). 
Proof. By multiplying equation (3.2a) by $\varphi_{h} \in \mathcal{V}_{h}$, integrating over $\Omega$, and using the Green's integration formula, we have:

$$
\begin{aligned}
\int_{\Omega} \varphi_{h} \frac{\partial u}{\partial t} \mathrm{~d} \Omega & +\int_{\Omega} \nabla \varphi_{h} \cdot \boldsymbol{\sigma} \nabla u \mathrm{~d} \Omega+\int_{\Omega} \varphi_{h} \boldsymbol{\beta} \cdot \nabla u \mathrm{~d} \Omega+\int_{\Omega} \varphi_{h} \kappa u \mathrm{~d} \Omega \\
& -\int_{\Gamma} \varphi_{h}(\boldsymbol{\sigma} \nabla u \cdot \mathbf{n}) \mathrm{d} \Gamma=\int_{\Omega} \varphi_{h} f \mathrm{~d} \Omega .
\end{aligned}
$$

Next, we consider the BC of equation (3.2b) multiplied by the function $\xi_{4}^{b} \varphi_{h}$, where $\xi_{4}^{b}$ is defined in equation (3.12), with $k=4$. By integrating this equation over $\Gamma_{b}$, for all $b=1, \ldots, N_{b}^{h}$, we obtain:

$$
\int_{\Gamma_{b}} \varphi_{h}\left(\frac{\xi}{\xi+\gamma h_{b}}\right)(\boldsymbol{\sigma} \nabla u \cdot \mathbf{n}) \mathrm{d} \Gamma+\int_{\Gamma_{b}} \varphi_{h}\left(\frac{\xi \gamma|\boldsymbol{\sigma}|}{\xi+\gamma h_{b}}\right) u \mathrm{~d} \Gamma=\int_{\Gamma_{b}} \varphi_{h}\left(\frac{\xi}{\xi+\gamma h_{b}}\right)(G+\gamma|\boldsymbol{\sigma}| g) \mathrm{d} \Gamma
$$

Similarly, by multiplying the BC of equation $(3.2 \mathrm{~b})$ by the function $\left(-\frac{1}{|\boldsymbol{\sigma}|} \xi_{3}^{b} \boldsymbol{\Phi}_{i n}^{*}\left(\varphi_{h}\right) \cdot \mathbf{n}\right)$, where $\xi_{3}^{b}$ is defined in equation (3.12), with $k=3$, and $\boldsymbol{\Phi}_{i n}^{*}(\cdot)$ in equation (3.8), and by integrating the resulting equation over $\Gamma_{b}$, for all $b=1, \ldots, N_{b}^{h}$, we have:

$$
\begin{aligned}
\int_{\Gamma_{b}} \boldsymbol{\Phi}_{i n}^{*}\left(\varphi_{h}\right) \cdot \mathbf{n}\left(-\frac{1}{|\boldsymbol{\sigma}|} \frac{h_{b}}{\xi+\gamma h_{b}}\right)(\boldsymbol{\sigma} \nabla u \cdot \mathbf{n}) \mathrm{d} & \Gamma+\int_{\Gamma_{b}} \boldsymbol{\Phi}_{i n}^{*}\left(\varphi_{h}\right) \cdot \mathbf{n}\left(-\frac{\gamma h_{b}}{\xi+\gamma h_{b}}\right) u \mathrm{~d} \Gamma \\
& =\int_{\Gamma_{b}} \boldsymbol{\Phi}_{i n}^{*}\left(\varphi_{h}\right) \cdot \mathbf{n}\left(-\frac{1}{|\boldsymbol{\sigma}|} \frac{h_{b}}{\xi+\gamma h_{b}}\right)(G+\gamma|\boldsymbol{\sigma}| g) \mathrm{d} \Gamma .
\end{aligned}
$$

Therefore, by summing side by side equations (3.34),(3.35), and(3.36), by noticing that $\sum_{b=1}^{N_{b}^{h}} \int_{\Gamma_{b}} \cdot \mathrm{d} \Gamma \equiv \int_{\Gamma} \cdot \mathrm{d} \Gamma$, and by using the definitions of equations (3.5) and (3.6), the thesis follows since $\int_{\Omega} \varphi_{h} \frac{\partial u_{h}}{\partial t} \mathrm{~d} \Omega+a_{h}\left(t ; u_{h}, \varphi_{h}\right)=$ $\mathcal{F}_{h}\left(t ; \varphi_{h}\right)$ for all $\varphi_{h} \in \mathcal{V}_{h}$.

In the following two lemmas we show the continuity and coercivity of the bilinear form $a_{h}(\cdot ; \cdot, \cdot)$ in equation (3.5) and the continuity the functional $\mathcal{F}_{h}(\cdot ; \cdot)$ in equation $(3.6)$ both in terms of the norm $\|\cdot\|_{h}$.

Lemma 3.9. Let $\boldsymbol{\sigma}_{0}:=\inf _{(\mathbf{x}, t) \in Q_{T}}\{\boldsymbol{\sigma}(\mathbf{x}, t)\}, \kappa_{0}:=\inf _{(\mathbf{x}, t) \in Q_{T}}\{\kappa(\mathbf{x}, t)\}$ and

$$
\bar{\alpha}:=\frac{\boldsymbol{\sigma}_{0}+\frac{1}{2} \inf _{Q_{T}}\{\boldsymbol{\beta} \cdot \mathbf{n}\} C_{\mathrm{tr}}^{2}-\frac{3}{2}\|\boldsymbol{\beta} \cdot \mathbf{n}\|_{\mathrm{L}^{\infty}\left(Q_{T}\right)} C_{\mathrm{tr}}^{2}}{\|\boldsymbol{\sigma}\|_{\mathrm{W}^{1, \infty}\left(Q_{T}\right)}} .
$$

Moreover, let the data of problem(3.4) satisfy the following conditions:

$$
\bar{\alpha}>0 \quad \text { and } \quad \kappa_{0}+\frac{1}{2} \inf _{Q_{T}}\{\boldsymbol{\beta} \cdot \mathbf{n}\} C_{\mathrm{tr}}^{2}-\frac{3}{2}\|\boldsymbol{\beta} \cdot \mathbf{n}\|_{\mathrm{L}^{\infty}\left(Q_{T}\right)} C_{\mathrm{tr}}^{2}>0 .
$$

In addition, let the penalty function $\xi$ satisfy the condition:

$$
\xi(\mathbf{x}, t)>h_{b} \chi_{\Gamma_{b}}(\mathbf{x}) \quad \forall(\mathbf{x}, t) \in \Sigma, \forall b=1, \ldots, N_{b}^{h},
$$

and its lower bound $\bar{\xi}_{0}$ satisfy the following one:

$$
\bar{\xi}_{0}>\frac{\bar{C}_{2}}{\bar{\alpha}}\left(\frac{\|\boldsymbol{\beta} \cdot \mathbf{n}\|_{\mathrm{L}^{\infty}\left(Q_{T}\right)}}{2 \boldsymbol{\sigma}_{0}}+\frac{\|\boldsymbol{\sigma}\|_{\mathrm{W}^{1, \infty}\left(Q_{T}\right)}}{2 \boldsymbol{\sigma}_{0}}\right),
$$


with $\bar{C}_{2}$ the constant associated to the trace inequality(3.16b). Then, there exist positive constants $M^{a}>0$ and $\alpha^{a}>0$, both independent of $h$, such that:

(i) $\left|a_{h}\left(t ; u_{h}, \varphi_{h}\right)\right| \leq M^{a}\left\|u_{h}\right\|_{h}\left\|\varphi_{h}\right\|_{h} \quad \forall u_{h}, \varphi_{h} \in \mathcal{V}_{h}$ and a.e. in $(0, T)$;

(ii) $a_{h}\left(t ; \varphi_{h}, \varphi_{h}\right) \geq \alpha^{a}\left\|\varphi_{h}\right\|_{h}^{2} \quad \forall \varphi_{h} \in \mathcal{V}_{h}$ and a.e. in $(0, T)$.

Proof.

(i) For $u_{h}, \varphi_{h} \in \mathcal{V}_{h}$ and by applying the triangle inequality to $\left|a_{h}\left(t ; u_{h}, \varphi_{h}\right)\right|$, we have:

$$
\begin{aligned}
\left|a_{h}\left(t ; u_{h}, \varphi_{h}\right)\right| \leq & \left|a_{\boldsymbol{\sigma}, \boldsymbol{\beta}, \kappa}\left(t ; u_{h}, \varphi_{h}\right)\right|+\sum_{b=1}^{N_{b}^{h}}\left[\left|\int_{\Gamma_{b}} \varphi_{h}\left(-\frac{\gamma h_{b}}{\xi+\gamma h_{b}}\right)\left(\boldsymbol{\sigma} \nabla u_{h} \cdot \mathbf{n}\right) \mathrm{d} \Gamma\right|\right. \\
& +\left|\int_{\Gamma_{b}}\left(\boldsymbol{\sigma} \nabla \varphi_{h} \cdot \mathbf{n}+\varphi_{h}(\boldsymbol{\beta} \cdot \mathbf{n}) \chi_{i n}\right)\left(-\frac{\gamma h_{b}}{\xi+\gamma h_{b}}\right) u_{h} \mathrm{~d} \Gamma\right|+\left|\int_{\Gamma_{b}} \varphi_{h}\left(\frac{|\boldsymbol{\sigma}| \xi \gamma}{\xi+\gamma h_{b}}\right) u_{h} \mathrm{~d} \Gamma\right| \\
& \left.+\left|\int_{\Gamma_{b}}\left(\boldsymbol{\sigma} \nabla \varphi_{h} \cdot \mathbf{n}+\varphi_{h}(\boldsymbol{\beta} \cdot \mathbf{n}) \chi_{i n}\right)\left(-\frac{h_{b}}{|\boldsymbol{\sigma}|\left(\xi+\gamma h_{b}\right)}\right)\left(\boldsymbol{\sigma} \nabla u_{h} \cdot \mathbf{n}\right) \mathrm{d} \Gamma\right|\right] .
\end{aligned}
$$

Moreover, by using the hypothesis(3.39), we have $\xi_{1}^{b}(\mathbf{x}, t) \leq \xi_{2}^{b}(\mathbf{x}, t)$. Then, in combination with the bounds(3.14), we have:

$$
\begin{aligned}
\left|a_{h}\left(t ; u_{h}, \varphi_{h}\right)\right| \leq & \|\boldsymbol{\sigma}\|_{\mathrm{W}^{1, \infty}\left(Q_{T}\right)}\left|\varphi_{h}\right|_{\mathrm{H}^{1}(\Omega)}\left|u_{h}\right|_{\mathrm{H}^{1}(\Omega)}+\|\boldsymbol{\beta}\|_{\mathrm{L}^{\infty}\left(Q_{T}\right)}\left\|\varphi_{h}\right\|_{\mathrm{L}^{2}(\Omega)}\left|u_{h}\right|_{\mathrm{H}^{1}(\Omega)} \\
& +\|\kappa\|_{\mathrm{L}^{\infty}\left(Q_{T}\right)}\left\|\varphi_{h}\right\|_{\mathrm{L}^{2}(\Omega)}\left\|u_{h}\right\|_{\mathrm{L}^{2}(\Omega)} \\
& +\sum_{b=1}^{N_{b}^{h}}\left[\|\boldsymbol{\sigma}\|_{\mathrm{W}^{1, \infty}\left(Q_{T}\right)}\left\|\zeta \varphi_{h}\right\|_{\mathrm{L}^{2}\left(\Gamma_{b}\right)}\left\|\sqrt{\frac{h_{b}}{\xi}} \sqrt{\frac{\gamma h_{b}}{\xi+\gamma h_{b}}} \nabla u_{h} \cdot \mathbf{n}\right\|_{\mathrm{L}^{2}\left(\Gamma_{b}\right)}\right. \\
& +\|\boldsymbol{\sigma}\|_{\mathrm{W}^{1, \infty}\left(Q_{T}\right)}\left\|\sqrt{\frac{h_{b}}{\xi}} \sqrt{\frac{\gamma h_{b}}{\xi+\gamma h_{b}}} \nabla \varphi_{h} \cdot \mathbf{n}\right\|_{\mathrm{L}^{2}\left(\Gamma_{b}\right)}\left\|\zeta u_{h}\right\|_{\mathrm{L}^{2}\left(\Gamma_{b}\right)} \\
& +\|\boldsymbol{\beta} \cdot \mathbf{n}\|_{\mathrm{L}^{\infty}\left(Q_{T}\right)}\left\|\zeta \varphi_{h}\right\|_{\mathrm{L}^{2}\left(\Gamma_{b}\right)}\left\|\zeta u_{h}\right\|_{\mathrm{L}^{2}\left(\Gamma_{b}\right)}+|\boldsymbol{\sigma}|\left\|\zeta \varphi_{h}\right\|_{\mathrm{L}^{2}\left(\Gamma_{b}\right)}\left\|\zeta u_{h}\right\|_{\mathrm{L}^{2}\left(\Gamma_{b}\right)} \\
& +\frac{\|\boldsymbol{\sigma}\|_{\mathrm{W}^{1, \infty}\left(Q_{T}\right)}^{2}}{|\boldsymbol{\sigma}|}\left\|\nabla \varphi_{h} \cdot \mathbf{n}\right\|_{\mathrm{L}^{2}\left(\Gamma_{b}\right)}\left\|\nabla u_{h} \cdot \mathbf{n}\right\|_{\mathrm{L}^{2}\left(\Gamma_{b}\right)} \\
& \left.+\frac{\|\boldsymbol{\sigma}\|_{\mathrm{W}^{1, \infty}\left(Q_{T}\right)}}{|\boldsymbol{\sigma}|}\|\boldsymbol{\beta} \cdot \mathbf{n}\|_{\mathrm{L}^{\infty}\left(Q_{T}\right)} \frac{h}{\bar{\xi}_{0}}\left\|\varphi_{h}\right\|_{\mathrm{L}^{2}\left(\Gamma_{b}\right)}\left\|\nabla u_{h} \cdot \mathbf{n}\right\|_{\mathrm{L}^{2}\left(\Gamma_{b}\right)}\right] .
\end{aligned}
$$

By using the Hölder's inequality,(3.16),(3.25b), and(3.21), we have:

$$
\begin{aligned}
\left|a_{h}\left(t ; u_{h}, \varphi_{h}\right)\right| \leq & \|\boldsymbol{\sigma}\|_{\mathrm{W}^{1, \infty}\left(Q_{T}\right)}\left|\varphi_{h}\right|_{\mathrm{H}^{1}(\Omega)}\left|u_{h}\right|_{\mathrm{H}^{1}(\Omega)}+\|\boldsymbol{\beta}\|_{\mathrm{L}^{\infty}\left(Q_{T}\right)} \frac{\sqrt{2}}{C_{\Omega}}\left\|\varphi_{h}\right\|_{h}\left|u_{h}\right|_{\mathrm{H}^{1}(\Omega)} \\
& +\|\kappa\|_{\mathrm{L}^{\infty}\left(Q_{T}\right)} \frac{2}{C_{\Omega}^{2}}\left\|\varphi_{h}\right\|_{h}\left\|u_{h}\right\|_{h} \\
& +\|\boldsymbol{\sigma}\|_{\mathrm{W}^{1, \infty}\left(Q_{T}\right)}\left\|\varphi_{h}\right\|_{h} \sqrt{\frac{2 \bar{C}_{2} C_{\Omega_{B}}}{\bar{\xi}_{0}}}\left\|u_{h}\right\|_{h}+\|\boldsymbol{\sigma}\|_{\mathrm{W}^{1, \infty}\left(Q_{T}\right)} \sqrt{\frac{2 \bar{C}_{2} C_{\Omega_{B}}}{\bar{\xi}_{0}}\left\|\varphi_{h}\right\|_{h}\left\|u_{h}\right\|_{h}} \\
& +\|\boldsymbol{\beta} \cdot \mathbf{n}\|_{\mathrm{L}^{\infty}\left(Q_{T}\right)}\left\|\varphi_{h}\right\|_{h}\left\|u_{h}\right\|_{h}+|\boldsymbol{\sigma}|\left\|\varphi_{h}\right\|_{h}\left\|u_{h}\right\|_{h}+\frac{\|\boldsymbol{\sigma}\|_{\mathrm{W}^{1, \infty}\left(Q_{T}\right)}^{2}}{|\boldsymbol{\sigma}|} \frac{\bar{C}_{2}}{\bar{\xi}_{0}} C_{\Omega_{B}}\left\|\varphi_{h}\right\|_{h}\left\|u_{h}\right\|_{h} \\
& +\frac{\|\boldsymbol{\sigma}\|_{\mathrm{W}^{1, \infty}\left(Q_{T}\right)}}{|\boldsymbol{\sigma}|}\|\boldsymbol{\beta} \cdot \mathbf{n}\|_{\mathrm{L}^{\infty}\left(Q_{T}\right)} \frac{C_{\Omega_{B}} \sqrt{\bar{C}_{1} \bar{C}_{2}}}{\bar{\xi}_{0}}\left\|\varphi_{h}\right\|_{h}\left\|u_{h}\right\|_{h} .
\end{aligned}
$$


Since $|\cdot|_{\mathrm{H}^{1}(\Omega)} \leq\|\cdot\|_{h}$, the continuity of $a_{h}(\cdot ; \cdot, \cdot)$ follows by setting:

$$
\begin{aligned}
M^{a}:=\max \{ & \|\boldsymbol{\sigma}\|_{\mathrm{W}^{1, \infty}\left(Q_{T}\right)} \max \left\{1, \sqrt{\frac{2 \bar{C}_{2} C_{\Omega_{B}}}{\bar{\xi}_{0}}}, \frac{\|\boldsymbol{\sigma}\|_{\mathrm{W}^{1, \infty}\left(Q_{T}\right)}}{|\boldsymbol{\sigma}|} \frac{\bar{C}_{2}}{\bar{\xi}_{0}} C_{\Omega_{B}}, \frac{\|\boldsymbol{\beta} \cdot \mathbf{n}\|_{\mathrm{L}^{\infty}\left(Q_{T}\right)}}{|\boldsymbol{\sigma}|} \frac{C_{\Omega_{B}} \sqrt{\bar{C}_{1} \bar{C}_{2}}}{\bar{\xi}_{0}}\right\}, \\
& \left.\|\boldsymbol{\beta}\|_{\mathrm{L}^{\infty}\left(Q_{T}\right)} \frac{\sqrt{2}}{C_{\Omega}},\|\kappa\|_{\mathrm{L}^{\infty}\left(Q_{T}\right)} \frac{2}{C_{\Omega}^{2}},\|\boldsymbol{\beta} \cdot \mathbf{n}\|_{\mathrm{L}^{\infty}\left(Q_{T}\right)},|\boldsymbol{\sigma}|\right\} .
\end{aligned}
$$

(ii) To show the coercivity of the bilinear form, we firstly focus on its boundary terms:

$$
\begin{aligned}
a_{\gamma}\left(t ; \varphi_{h}, \varphi_{h}\right):= & \sum_{b=1}^{N_{b}^{h}} \int_{\Gamma_{b}} \varphi_{h}\left(-\frac{\gamma h_{b}}{\xi+\gamma h_{b}}\right)\left(\boldsymbol{\sigma} \nabla \varphi_{h} \cdot \mathbf{n}\right) \mathrm{d} \Gamma+\int_{\Gamma_{b}}\left(\boldsymbol{\Phi}_{i n}^{*}\left(\varphi_{h}\right) \cdot \mathbf{n}\right)\left(-\frac{\gamma h_{b}}{\xi+\gamma h_{b}}\right) \varphi_{h} \mathrm{~d} \Gamma \\
& +\int_{\Gamma_{b}} \varphi_{h}\left(\frac{|\boldsymbol{\sigma}| \xi \gamma}{\xi+\gamma h_{b}}\right) \varphi_{h} \mathrm{~d} \Gamma+\int_{\Gamma_{b}}\left(\boldsymbol{\Phi}_{i n}^{*}\left(\varphi_{h}\right) \cdot \mathbf{n}\right)\left(-\frac{h_{b}}{|\boldsymbol{\sigma}|\left(\xi+\gamma h_{b}\right)}\right)\left(\boldsymbol{\sigma} \nabla \varphi_{h} \cdot \mathbf{n}\right) \mathrm{d} \Gamma .
\end{aligned}
$$

We have:

$$
\begin{aligned}
& a_{\gamma}\left(t ; \varphi_{h}, \varphi_{h}\right) \geq \sum_{b=1}^{N_{b}^{h}} \underbrace{(-2)\|\boldsymbol{\sigma}\|_{\mathrm{W}^{1, \infty}\left(Q_{T}\right)}\left\|\xi_{1}^{b}\right\|_{\mathrm{L}^{\infty}\left(\Gamma_{b}\right)}\left\|\varphi_{h}\right\|_{\mathrm{L}^{2}\left(\Gamma_{b}\right)}\left\|\nabla \varphi_{h} \cdot \mathbf{n}\right\|_{\mathrm{L}^{2}\left(\Gamma_{b}\right)}}_{(a)} \\
& -\underbrace{\frac{\|\boldsymbol{\beta} \cdot \mathbf{n}\|_{\mathrm{L}^{\infty}\left(Q_{T}\right)}}{\boldsymbol{\sigma}_{0}}\|\boldsymbol{\sigma}\|_{\mathrm{W}^{1, \infty}\left(Q_{T}\right)}\left\|\xi_{3}^{b}\right\|_{\mathrm{L}^{\infty}\left(\Gamma_{b}\right)}\left\|\varphi_{h}\right\|_{\mathrm{L}^{2}\left(\Gamma_{b}\right)}\left\|\nabla \varphi_{h} \cdot \mathbf{n}\right\|_{\mathrm{L}^{2}\left(\Gamma_{b}\right)}}_{(b)} \\
& +\|\boldsymbol{\sigma}\|_{\mathrm{W}^{1, \infty}\left(Q_{T}\right)}\left\|\zeta \varphi_{h}\right\|_{\mathrm{L}^{2}\left(\Gamma_{b}\right)}^{2}-\underbrace{\int_{\Gamma_{b}}(\boldsymbol{\beta} \cdot \mathbf{n}) \chi_{i n} \xi_{1}^{b} \varphi_{h}^{2} \mathrm{~d} \Gamma}_{(c)} \\
& -\underbrace{\frac{\|\boldsymbol{\sigma}\|_{\mathrm{W}^{1, \infty}\left(Q_{T}\right)}^{2}}{\boldsymbol{\sigma}_{0}}\left\|\xi_{3}^{b}\right\|_{\mathrm{L}^{\infty}\left(\Gamma_{b}\right)}\left\|\nabla \varphi_{h} \cdot \mathbf{n}\right\|_{\mathrm{L}^{2}\left(\Gamma_{b}\right)}^{2}}_{(d)} .
\end{aligned}
$$

The term $(a)$ is bounded using the Young's inequality $\iota v \leq \delta \iota^{2}+\frac{1}{4 \delta} v^{2}$ for $\iota, v \in \mathbb{R}$, for all $\delta>0$, where we set $\iota=\frac{h}{\bar{\xi}_{0}}\|\zeta\|_{\mathrm{L}^{\infty}\left(\Gamma_{b}\right)}\|\boldsymbol{\sigma}\|_{\mathrm{W}^{1, \infty}\left(Q_{T}\right)}^{1 / 2}\left\|\nabla \varphi_{h} \cdot \mathbf{n}\right\|_{\mathrm{L}^{2}\left(\Gamma_{b}\right)}$ and $v=2\|\zeta\|_{\mathrm{L}^{\infty}\left(\Gamma_{b}\right)}\|\boldsymbol{\sigma}\|_{\mathrm{W}^{1, \infty}\left(Q_{T}\right)}^{1 / 2}\left\|\varphi_{h}\right\|_{\mathrm{L}^{2}\left(\Gamma_{b}\right)}$. Then, by considering(3.16b), we have:

$$
\begin{aligned}
(a) & \geq-\sum_{b=1}^{N_{b}^{h}}\left\{\frac{1}{\delta} \frac{h^{2}}{\bar{\xi}_{0}^{2}}\|\zeta\|_{\mathrm{L}^{\infty}\left(\Gamma_{b}\right)}^{2}\|\boldsymbol{\sigma}\|_{\mathrm{W}^{1, \infty}\left(Q_{T}\right)}\left\|\nabla \varphi_{h} \cdot \mathbf{n}\right\|_{\mathrm{L}^{2}\left(\Gamma_{b}\right)}^{2}+\delta\|\zeta\|_{\mathrm{L}^{\infty}\left(\Gamma_{b}\right)}^{2}\|\boldsymbol{\sigma}\|_{\mathrm{W}^{1, \infty}\left(Q_{T}\right)}\left\|\varphi_{h}\right\|_{\mathrm{L}^{2}\left(\Gamma_{b}\right)}^{2}\right\} \\
& \geq-\sum_{b=1}^{N_{b}^{h}}\left\{\frac{1}{\delta} \frac{h}{\bar{\xi}_{0}^{2}}\|\zeta\|_{\mathrm{L}^{\infty}\left(\Gamma_{b}\right)}^{2}\|\boldsymbol{\sigma}\|_{\mathrm{W}^{1, \infty}\left(Q_{T}\right)} \bar{C}_{2}\left|\varphi_{h}\right|_{\mathrm{H}^{1}\left(T_{b}\right)}^{2}+\delta\|\boldsymbol{\sigma}\|_{\mathrm{W}^{1, \infty}\left(Q_{T}\right)}\left\|\zeta \varphi_{h}\right\|_{\mathrm{L}^{2}\left(\Gamma_{b}\right)}^{2}\right\} ;
\end{aligned}
$$


The term $(b)$ is bounded by applying the previous Young's inequality with $\iota=\left\|\varphi_{h}\right\|_{\mathrm{L}^{2}\left(\Gamma_{b}\right)}$, $v=\left\|\nabla \varphi_{h} \cdot \mathbf{n}\right\|_{\mathrm{L}^{2}\left(\Gamma_{b}\right)}$, and $\delta=\frac{1}{2}$, together with(3.16b), as:

$$
\begin{aligned}
(b) & \geq-\sum_{b=1}^{N_{b}^{h}} \frac{\|\boldsymbol{\beta} \cdot \mathbf{n}\|_{\mathrm{L}^{\infty}\left(Q_{T}\right)}}{\boldsymbol{\sigma}_{0}}\|\boldsymbol{\sigma}\|_{\mathrm{W}^{1, \infty}\left(Q_{T}\right)}\left\|\xi_{3}^{b}\right\|_{\mathrm{L}^{\infty}\left(\Gamma_{b}\right)}\left(\frac{\left\|\varphi_{h}\right\|_{\mathrm{L}^{2}\left(\Gamma_{b}\right)}^{2}}{2}+\frac{\left\|\nabla \varphi_{h} \cdot \mathbf{n}\right\|_{\mathrm{L}^{2}\left(\Gamma_{b}\right)}^{2}}{2}\right) \\
& \geq-\sum_{b=1}^{N_{b}^{h}} \frac{\|\boldsymbol{\beta} \cdot \mathbf{n}\|_{\mathrm{L}^{\infty}\left(Q_{T}\right)}}{\boldsymbol{\sigma}_{0}}\|\boldsymbol{\sigma}\|_{\mathrm{W}^{1, \infty}\left(Q_{T}\right)}\left(\frac{\bar{\xi}_{\infty}}{2 \bar{\xi}_{0}}\left\|\zeta \varphi_{h}\right\|_{\mathrm{L}^{2}\left(\Gamma_{b}\right)}^{2}-\left\|\frac{1}{\xi+\gamma h_{b}}\right\|_{\mathrm{L}^{\infty}(\Gamma)} \bar{C}_{2}\left|\varphi_{h}\right|_{\mathrm{H}^{1}\left(T_{b}\right)}^{2}\right) .
\end{aligned}
$$

As for term $(c)$, we simply observe that:

$$
(c) \geq-2 \sum_{b=1}^{N_{b}^{h}} \int_{\Gamma_{b}}(\boldsymbol{\beta} \cdot \mathbf{n}) \chi_{i n} \varphi_{h}^{2} \mathrm{~d} \Gamma \geq-2 \int_{\Gamma}(\boldsymbol{\beta} \cdot \mathbf{n}) \chi_{i n} \varphi_{h}^{2} \mathrm{~d} \Gamma .
$$

Finally, the term $(d)$ can be bounded similarly to term $(b)$, for which we have:

$$
(d) \geq-\sum_{b=1}^{N_{b}^{h}}\left\|\frac{1}{\xi+\gamma h_{b}}\right\|_{L^{\infty}(\Gamma)} \frac{\|\boldsymbol{\sigma}\|_{\mathrm{W}^{1, \infty}\left(Q_{T}\right)}^{2}}{\boldsymbol{\sigma}_{0}} \bar{C}_{2}\left|\varphi_{h}\right|_{\mathrm{H}^{1}\left(T_{b}\right)}^{2} .
$$

On the other hand, the hypothesis $\nabla \cdot \boldsymbol{\beta}=0$ a.e. in $\Omega$ yields the identity $\int_{\Omega} \varphi_{h} \boldsymbol{\beta} \cdot \nabla \varphi_{h} \mathrm{~d} \Omega=\frac{1}{2} \int_{\Gamma} \varphi_{h}^{2} \boldsymbol{\beta} \cdot \mathbf{n} \mathrm{d} \Gamma$. Then, using the trace inequality(3.26), we can bound the sum of $a_{\boldsymbol{\sigma}, \boldsymbol{\beta}, \kappa}\left(t ; \varphi_{h}, \varphi_{h}\right)$ and term (c) as:

$$
\begin{aligned}
a_{\boldsymbol{\sigma}, \boldsymbol{\beta}, \kappa}\left(t ; \varphi_{h}, \varphi_{h}\right)+(c) \geq & \boldsymbol{\sigma}_{0}\left|\varphi_{h}\right|_{\mathrm{H}^{1}(\Omega)}^{2}+\kappa_{0}\left\|\varphi_{h}\right\|_{\mathrm{L}^{2}(\Omega)}^{2}+\frac{1}{2} \int_{\Gamma}(\boldsymbol{\beta} \cdot \mathbf{n})\left[\chi_{\text {in }}+\chi_{\text {out }}\right] \varphi_{h}^{2} \mathrm{~d} \Gamma \\
& -2 \int_{\Gamma}(\boldsymbol{\beta} \cdot \mathbf{n}) \chi_{i n} \varphi_{h}^{2} \mathrm{~d} \Gamma \\
\geq & \boldsymbol{\sigma}_{0}\left|\varphi_{h}\right|_{\mathrm{H}^{1}(\Omega)}^{2}+\kappa_{0}\left\|\varphi_{h}\right\|_{\mathrm{L}^{2}(\Omega)}^{2}+\left(\frac{1}{2} \inf _{Q_{T}}\{\boldsymbol{\beta} \cdot \mathbf{n}\}-\frac{3}{2}\|\boldsymbol{\beta} \cdot \mathbf{n}\|_{\mathrm{L}^{\infty}\left(Q_{T}\right)}\right)\left\|\varphi_{h}\right\|_{\mathrm{L}^{2}(\Gamma)}^{2} \\
\geq & \left(\boldsymbol{\sigma}_{0}+\frac{1}{2} \inf _{Q_{T}}\{\boldsymbol{\beta} \cdot \mathbf{n}\}-\frac{3}{2}\|\boldsymbol{\beta} \cdot \mathbf{n}\|_{\mathrm{L}^{\infty}\left(Q_{T}\right)}\right) C_{\mathrm{tr}}^{2}\left|\varphi_{h}\right|_{\mathrm{H}^{1}(\Omega)}^{2} \\
& +\left(\kappa_{0}+\frac{1}{2} \inf _{Q_{T}}\{\boldsymbol{\beta} \cdot \mathbf{n}\}-\frac{3}{2}\|\boldsymbol{\beta} \cdot \mathbf{n}\|_{\mathrm{L}^{\infty}\left(Q_{T}\right)}\right) C_{\mathrm{tr}}^{2}\left\|\varphi_{h}\right\|_{\mathrm{L}^{2}(\Omega)}^{2} .
\end{aligned}
$$

By combining(3.48),(3.44),(3.45), and(3.46), under the hypothesis(3.38), we have:

$$
\begin{aligned}
a_{h}\left(t ; \varphi_{h}, \varphi_{h}\right)= & a_{\boldsymbol{\sigma}, \boldsymbol{\beta}, \kappa}\left(t ; \varphi_{h}, \varphi_{h}\right)+a_{\gamma}\left(t ; \varphi_{h}, \varphi_{h}\right) \\
\geq & \left(\boldsymbol{\sigma}_{0}+\frac{1}{2} \inf _{Q_{T}}\{\boldsymbol{\beta} \cdot \mathbf{n}\} C_{\mathrm{tr}}^{2}-\frac{3}{2}\|\boldsymbol{\beta} \cdot \mathbf{n}\|_{\mathrm{L}^{\infty}\left(Q_{T}\right)} C_{\mathrm{tr}}^{2}\right)\left|\varphi_{h}\right|_{\mathrm{H}^{1}(\Omega)}^{2} \\
& -\frac{1}{\delta} \frac{h}{\bar{\xi}_{0}^{2}}\|\zeta\|_{\mathrm{L}^{\infty}\left(\Gamma_{b}\right)}^{2}\|\boldsymbol{\sigma}\|_{\mathrm{W}^{1, \infty}\left(Q_{T}\right)} \bar{C}_{2}\left|\varphi_{h}\right|_{\mathrm{H}^{1}(\Omega)}^{2}-\delta\|\boldsymbol{\sigma}\|_{\mathrm{W}^{1, \infty}\left(Q_{T}\right)} \sum_{b=1}^{N_{b}^{h}}\left\|\zeta \varphi_{h}\right\|_{\mathrm{L}^{2}\left(\Gamma_{b}\right)}^{2} \\
& -\frac{\|\boldsymbol{\beta} \cdot \mathbf{n}\|_{\mathrm{L}^{\infty}\left(Q_{T}\right)}\|\boldsymbol{\sigma}\|_{\mathrm{W}^{1, \infty}\left(Q_{T}\right)} \frac{\bar{\xi}_{\infty}}{\bar{\xi}_{0}} \sum_{b=1}^{N_{b}^{h}}\left\|\zeta \varphi_{h}\right\|_{\mathrm{L}^{2}\left(\Gamma_{b}\right)}^{2}}{} \\
& -\frac{\|\boldsymbol{\beta} \cdot \mathbf{n}\|_{\mathrm{L}^{\infty}\left(Q_{T}\right)}\|\boldsymbol{\sigma}\|_{\mathrm{W}^{1, \infty}\left(Q_{T}\right)}\left\|\frac{1}{\xi \boldsymbol{\sigma}_{0}}\right\| \gamma h_{b} \|_{\mathrm{L}^{\infty}(\Gamma)} \bar{C}_{2}\left|\varphi_{h}\right|_{\mathrm{H}^{1}(\Omega)}^{2} \cdot}{} \\
& +\|\boldsymbol{\sigma}\|_{\mathrm{W}^{1, \infty}\left(Q_{T}\right)} \sum_{b=1}^{N_{b}^{h}}\left\|\zeta \varphi_{h}\right\|_{\mathrm{L}^{2}\left(\Gamma_{b}\right)}^{2}-\left\|\frac{1}{\xi+\gamma h_{b}}\right\|_{\mathrm{L}^{\infty}(\Gamma)} \frac{\|\boldsymbol{\sigma}\|_{\mathrm{W}^{1, \infty}\left(Q_{T}\right)}^{2} \bar{C}_{2}\left|\varphi_{h}\right|_{\mathrm{H}^{1}(\Omega)}^{2}}{\boldsymbol{\sigma}_{0}}
\end{aligned}
$$


Introducing the constant $\bar{\alpha}>0$ of(3.37) into(3.49); we obtain:

$$
\begin{gathered}
a_{h}\left(t ; \varphi_{h}, \varphi_{h}\right) \geq\|\boldsymbol{\sigma}\|_{\mathrm{W}^{1, \infty}\left(Q_{T}\right)} \bar{\alpha}\left|\varphi_{h}\right|_{\mathrm{H}^{1}(\Omega)}^{2} \\
-\|\boldsymbol{\sigma}\|_{\mathrm{W}^{1, \infty}\left(Q_{T}\right)} \bar{C}_{2}\left[\frac{1}{\delta} \frac{h}{\bar{\xi}_{0}^{2}}\|\zeta\|_{\mathrm{L}^{\infty}(\Gamma)}^{2}+\left\|\frac{1}{\xi+\gamma h_{b}}\right\|_{\mathrm{L}^{\infty}(\Gamma)}\left(\frac{\|\boldsymbol{\beta} \cdot \mathbf{n}\|_{\mathrm{L}^{\infty}\left(Q_{T}\right)}}{2 \boldsymbol{\sigma}_{0}}+\frac{\|\boldsymbol{\sigma}\|_{\mathrm{W}^{1, \infty}\left(Q_{T}\right)}}{2 \boldsymbol{\sigma}_{0}}\right)\right]\left|\varphi_{h}\right|_{\mathrm{H}^{1}(\Omega)}^{2} \\
+\|\boldsymbol{\sigma}\|_{\mathrm{W}^{1, \infty}\left(Q_{T}\right)}\left(1-\delta-\frac{\|\boldsymbol{\beta} \cdot \mathbf{n}\|_{\mathrm{L}^{\infty}\left(Q_{T}\right)} \bar{\xi}_{\infty}}{2 \boldsymbol{\sigma}_{0}}\right) \sum_{b=1}^{N_{b}^{h}}\left\|\zeta \varphi_{h}\right\|_{\mathrm{L}^{2}\left(\Gamma_{b}\right)}^{2} \\
\geq\|\boldsymbol{\sigma}\|_{\mathrm{W}^{1, \infty}\left(Q_{T}\right)} \bar{\alpha}\|\zeta\|_{\mathrm{L}^{\infty}\left(\Gamma_{b}\right)}^{2}\left[\left(\underset{\mathbf{x} \in \Gamma}{\operatorname{essinf}} \frac{1}{\gamma}\right)\left(1-\frac{\bar{C}_{2}}{\bar{\alpha}} \frac{1}{\bar{\xi}_{0}}\left(\frac{\|\boldsymbol{\beta} \cdot \mathbf{n}\|_{\mathrm{L}^{\infty}\left(Q_{T}\right)}}{2 \boldsymbol{\sigma}_{0}}+\frac{\|\boldsymbol{\sigma}\|_{\mathrm{W}^{1, \infty}\left(Q_{T}\right)}}{2 \boldsymbol{\sigma}_{0}}\right)\right)\right. \\
\left.+\frac{h}{\bar{\xi}_{\infty}}\left(1-\frac{\bar{C}_{2}}{\bar{\alpha}} \frac{1}{\delta} \frac{\bar{\xi}_{\infty}}{\bar{\xi}_{0}^{2}}\right)\right]\left|\varphi_{h}\right|_{\mathrm{H}^{1}(\Omega)}^{2}+\|\boldsymbol{\sigma}\|_{\mathrm{W}^{1, \infty}\left(Q_{T}\right)}\left(1-\delta-\frac{\|\boldsymbol{\beta} \cdot \mathbf{n}\|_{\mathrm{L}^{\infty}\left(Q_{T}\right)}}{2 \boldsymbol{\sigma}_{0}} \frac{\bar{\xi}_{\infty}}{\bar{\xi}_{0}}\right) \sum_{b=1}^{N_{b}^{h}}\left\|\zeta \varphi_{h}\right\|_{\mathrm{L}^{2}\left(\Gamma_{b}\right)}^{2} .
\end{gathered}
$$

By the hypothesis(3.40) on $\bar{\xi}_{0}$ and selecting $\delta$ such that $\frac{\bar{C}_{2}}{\bar{\alpha}} \frac{\bar{\xi}_{\infty}}{\bar{\xi}_{0}^{2}}<\delta<1-\frac{\|\boldsymbol{\beta} \cdot \mathbf{n}\|_{L_{\infty}\left(Q_{T}\right)}}{2 \sigma_{0}} \frac{\bar{\xi}_{\infty}}{\bar{\xi}_{0}}$, the coercivity follows with constant:

$$
\alpha^{a}:=\|\boldsymbol{\sigma}\|_{\mathrm{W}^{1, \infty}\left(Q_{T}\right)} \min \left\{\bar{\alpha}\left(1-\frac{\bar{C}_{2}}{\bar{\alpha}} \frac{1}{\delta} \frac{\bar{\xi}_{\infty}}{\bar{\xi}_{0}^{2}}\right),\left(1-\delta-\frac{\|\boldsymbol{\beta} \cdot \mathbf{n}\|_{\mathrm{L}^{\infty}\left(Q_{T}\right)}}{2 \boldsymbol{\sigma}_{0}} \frac{\bar{\xi}_{\infty}}{\bar{\xi}_{0}}\right)\right\}>0 .
$$

Lemma 3.10. Under the hypotheses of Lemma 3.9, there exists a positive constant $M_{h}^{\mathcal{F}}$, dependent on $h$ and on the data of the problem, such that:

$$
\left|\mathcal{F}_{h}\left(t ; \varphi_{h}\right)\right| \leq M_{h}^{\mathcal{F}}\left\|\varphi_{h}\right\|_{h} \quad \forall \varphi_{h} \in \mathcal{V}_{h} \text { and a.e. in }(0, T)
$$

Proof. The continuity of the functional $\mathcal{F}_{h}$ follows by the triangle and Schwarz's inequalities and by apply$\operatorname{ing}(3.14)$ :

$$
\begin{aligned}
\left|\mathcal{F}_{h}\left(t ; \varphi_{h}\right)\right| \leq & \left|\int_{\Omega} \varphi_{h} f \mathrm{~d} \Omega\right|+\mid \sum_{b=1}^{N_{b}^{h}}\left[\int_{\Gamma_{b}}\left(\boldsymbol{\Phi}_{i n}^{*}\left(\varphi_{h}\right) \cdot \mathbf{n}\right)\left(-\frac{\gamma h_{b}}{\xi+\gamma h_{b}}\right) g \mathrm{~d} \Gamma+\int_{\Gamma_{b}} \varphi_{h}\left(\frac{|\boldsymbol{\sigma}| \xi \gamma}{\xi+\gamma h_{b}}\right) g \mathrm{~d} \Gamma\right. \\
& \left.+\int_{\Gamma_{b}}\left(\boldsymbol{\Phi}_{i n}^{*}\left(\varphi_{h}\right) \cdot \mathbf{n}\right)\left(-\frac{h_{b}}{|\boldsymbol{\sigma}|\left(\xi+\gamma h_{b}\right)}\right) G \mathrm{~d} \Gamma+\int_{\Gamma_{b}} \varphi_{h}\left(\frac{\xi}{\xi+\gamma h_{b}}\right) G \mathrm{~d} \Gamma\right] \mid \\
\leq & \left\|\varphi_{h}\right\|_{\mathrm{L}^{2}(\Omega)}\|f\|_{\mathrm{L}^{\infty}\left(0, T ; \mathrm{L}^{2}(\Omega)\right)}+\left|\int_{\Gamma} \varphi_{h} G \mathrm{~d} \Gamma\right| \\
& +\sum_{b=1}^{N_{b}^{h}}\left[2\|\boldsymbol{\sigma}\|_{\mathrm{W}^{1, \infty}\left(Q_{T}\right)}\|g\|_{\mathrm{L}^{2}\left(\Gamma_{b}\right)}\left\|\nabla \varphi_{h} \cdot \mathbf{n}\right\|_{\mathrm{L}^{2}\left(\Gamma_{b}\right)}^{2}\right. \\
& +\sqrt{\frac{2 h_{b}}{\bar{\xi}_{0}}\|\boldsymbol{\beta} \cdot \mathbf{n}\|_{\mathrm{L}^{\infty}\left(Q_{T}\right)}\|g\|_{\mathrm{L}^{2}\left(\Gamma_{b}\right)}\left\|\zeta \varphi_{h}\right\|_{\mathrm{L}^{2}\left(\Gamma_{b}\right)}} \\
& +|\boldsymbol{\sigma}| \sqrt{\frac{\bar{\xi}_{\infty}}{h_{b}}}\|g\|_{\mathrm{L}^{2}\left(\Gamma_{b}\right)}\left\|\zeta \varphi_{h}\right\|_{\mathrm{L}^{2}\left(\Gamma_{b}\right)}+\frac{\|\boldsymbol{\sigma}\|_{\mathrm{W}^{1, \infty}\left(Q_{T}\right)}}{|\boldsymbol{\sigma}|} \frac{h_{b}}{\bar{\xi}_{0}}\|G\|_{\mathrm{L}^{2}\left(\Gamma_{b}\right)}\left\|\nabla \varphi_{h} \cdot \mathbf{n}\right\|_{\mathrm{L}^{2}\left(\Gamma_{b}\right)} \\
& \left.+\frac{\|\boldsymbol{\beta} \cdot \mathbf{n}\|_{\mathrm{L}^{\infty}\left(Q_{T}\right)}}{|\boldsymbol{\sigma}|} \frac{h_{b}}{\bar{\xi}_{0}}\|G\|_{\mathrm{L}^{2}\left(\Gamma_{b}\right)}\left\|\varphi_{h}\right\|_{\mathrm{L}^{2}\left(\Gamma_{b}\right)}\right] .
\end{aligned}
$$


Moreover, by using(3.16),(3.21),(3.25a), and(3.25b), we obtain the thesis by defining the continuity constant $M_{h}^{\mathcal{F}}$ as:

$$
\begin{aligned}
& M_{h}^{\mathcal{F}}:=\frac{\sqrt{2}}{C_{\Omega}}\|f\|_{\mathrm{L}^{\infty}\left(0, T ; \mathrm{L}^{2}(\Omega)\right)} \\
& +\left(\frac{\sqrt{2} C_{\mathrm{tr}}}{C_{\Omega}}+\frac{\|\boldsymbol{\sigma}\|_{\mathrm{W}^{1, \infty}\left(Q_{T}\right)}}{|\boldsymbol{\sigma}|} \sqrt{\frac{C_{\Omega_{B} \bar{C}_{2}}}{\bar{\xi}_{0}}}+\frac{\|\boldsymbol{\beta} \cdot \mathbf{n}\|_{\mathrm{L}^{\infty}\left(Q_{T}\right)}}{|\boldsymbol{\sigma}|} \sqrt{\frac{C_{\Omega_{B}} \bar{C}_{1}}{\bar{\xi}_{0}}}\right)\|G\|_{\mathrm{L}^{\infty}\left(0, T ; \mathrm{L}^{2}(\partial \Omega)\right)} \\
& +\left(\sqrt{2}\|\boldsymbol{\beta} \cdot \mathbf{n}\|_{\mathrm{L}^{\infty}\left(Q_{T}\right)}+\frac{1}{\sqrt{h}}\left(2\|\boldsymbol{\sigma}\|_{\mathrm{W}^{1, \infty}\left(Q_{T}\right)} \sqrt{C_{\Omega_{B}} \bar{C}_{2}}+|\boldsymbol{\sigma}| \sqrt{\overline{\xi_{\infty}}}\right)\right)\|g\|_{\mathrm{L}^{\infty}\left(0, T ; \mathrm{L}^{2}(\partial \Omega)\right)} .
\end{aligned}
$$

Remark 3.11. The constant $M_{h}^{\mathcal{F}}$ of equation (3.53) depends on the mesh size $h$ and actually tends to infinity as $h \rightarrow 0$. This result is expected, since analogous results are obtained for the weak imposition of the Dirichlet BCs [27] and follows from the fact that we use a mesh dependent norm $\|\cdot\|_{h}$. A continuity constant $\widetilde{M}^{\mathcal{F}}$ independent of $h$ for the functional $\mathcal{F}_{h}(t ; \cdot)$ can however be ensured for boundary data $g$ and $G$ sufficiently regular, i.e. such that their extensions to the domain $\Omega$ are well-defined, as well as their corresponding $\|\cdot\|_{h}$ norms; see [31].

The semi-discetized FE solution of problem(3.4) obtained by using the Nitsche's method, can be expressed, for each time $t \in(0, T)$, by using the basis functions $\varphi_{h, i}$ of the FE space $\mathcal{V}_{h}$ of equation (3.3) as $u_{h}(\mathbf{x}, t):=\sum_{i=1}^{N^{(h)}} d_{i}(t) \varphi_{h, i}(\mathbf{x})$, where $N^{(h)}:=\operatorname{dim} \mathcal{V}_{h}$. By substituting the expression of $u_{h}$ in equation (3.4), we obtain a system of linear ordinary differential equations in the unknown vector of functions $\boldsymbol{d}:=\left(d_{1}, \ldots, d_{N^{(h)}}\right):(0, T) \rightarrow \mathbb{R}^{N^{(h)}}$, which reads:

$$
\left\{\begin{array}{l}
\boldsymbol{M} \dot{\boldsymbol{d}}(t)+\boldsymbol{A}(t) \boldsymbol{d}(t)=\boldsymbol{F}(t) \quad \text { for } t \in(0, T) \\
\boldsymbol{M d}(0)=\widetilde{\boldsymbol{u}}_{0}
\end{array}\right.
$$

where $\boldsymbol{M}$ and $\boldsymbol{A}$ are the mass and stiffness matrices, defined as $\boldsymbol{M}_{i, j}:=\left(\varphi_{h, j}, \varphi_{h, i}\right)$ and $\boldsymbol{A}_{i, j}(t):=$ $a_{h}\left(t ; \varphi_{h, j}, \varphi_{h, i}\right)$, for all $i, j=1, \ldots, N^{(h)}$, respectively. Moreover, $\boldsymbol{F}(t)$ is a vector whose components are $\boldsymbol{F}_{i}(t):=\mathcal{F}_{h}\left(t ; \varphi_{h, i}\right)$ and the vector $\widetilde{\boldsymbol{u}}_{0}$ is such that $\widetilde{\boldsymbol{u}}_{0, i}:=\left(\widetilde{u}_{0}, \varphi_{h, i}\right)$, with $\widetilde{u}_{0}$ being the $\mathrm{L}^{2}$ projection of $u_{0}$ onto $\mathcal{V}_{h}$. By definition, the matrix $\boldsymbol{M}$ is symmetric and positive definite; moreover, by the coercivity of the bilinear form $a_{h}(t ; \cdot, \cdot)$ established in Lemma 3.9 (ii), also the matrix $\boldsymbol{A}$ is positive definite. From the continuity of both $a_{h}(t ; \cdot, \cdot)$ and $\mathcal{F}_{h}$ shown in Lemmas 3.9 and 3.10, the existence and uniqueness of the solution $\boldsymbol{d}$ of system (3.54), and consequently of the semi-discrete solution $u_{h}$, follows from the theory of ordinary differential equations $[12,26,27]$.

Remark 3.12. We remark that the condition (3.40) established in Lemma 3.9 is necessary to yield the well posedness of problem (3.4). Indeed, choosing values of $\xi=\xi(\mathbf{x}, t)$ which do not satisfy this condition leads the coercivity of the bilinear form $a_{h}(\cdot ; \cdot, \cdot)$ to not hold any more. We remark that the explicit expression for the lower bound of $\bar{\xi}_{0}$ in equation (3.40) depends on both the data of the problem $(\boldsymbol{\sigma}, \boldsymbol{\beta}$ and $\Omega$ ) and on the degree of the finite element space $k$ through the constant $\bar{C}_{2}$.

\subsection{The full discrete problem: the Nitsche's- $\theta$ method}

Let $N_{T} \in \mathbb{N}$ be a given integer and let us uniformly partition the time interval $(0, T)$ into a set of subintervals $\left\{\left[t^{n}, t^{n+1}\right)\right\}_{n=0}^{N_{T}-1}$ of size $\Delta t:=T / N_{T}$, with $t^{n}:=n \Delta t$. By applying the $\theta$-method [25] to the semi-discrete problem (3.4), this yields, for $n=1, \ldots, N_{T}$ :

$$
\text { find } u_{h}^{n} \in \mathcal{V}_{h}: \quad\left\{\begin{array}{l}
\frac{1}{\Delta t}\left(u_{h}^{n}-u_{h}^{n-1}, \varphi_{h}\right)+a_{h}\left(t_{\theta}^{n} ; u_{h, \theta}^{n}, \varphi_{h}\right)=\mathcal{F}_{h}\left(t_{\theta}^{n} ; \varphi_{h}\right) \quad \forall \varphi_{h} \in \mathcal{V}_{h}, \\
\widetilde{u}_{h}^{0}=\widetilde{u}_{0} ;
\end{array}\right.
$$


where, for $\theta \in[0,1]$, we have considered $t_{\theta}^{n}:=\theta t^{n}+(1-\theta) t^{n-1}, u_{h, \theta}^{n}:=\theta u_{h}^{n}+(1-\theta) u_{h}^{n-1}$, being $u_{h}^{n}$ the approximation of $u_{h}\left(\mathbf{x}, t^{n}\right)$. Moreover, for $\widetilde{u}_{0} \in \mathcal{V}_{h}$, the $\mathrm{L}^{2}$ projection of $u_{0}$ onto $\mathcal{V}_{h}$, we have from [26]:

$$
\left\|u_{0}-\widetilde{u}_{h}^{0}\right\|_{L^{2}(\Omega)}+h\left\|u_{0}-\widetilde{u}_{h}^{0}\right\|_{\mathrm{H}^{1}(\Omega)} \leq C h\left\|u_{0}\right\|_{\mathrm{H}^{1}(\Omega)} .
$$

In the following, we will show that equation (3.55) is unconditionally stable with respect to the $\mathrm{L}^{2}$ norm provided that $\theta \in[1 / 2,1]$, while is conditionally stable for $\theta \in[0,1 / 2)$.

Theorem 3.13. Let us consider problem (3.55) and a regular family of quasi-uniform triangulations $\left\{\mathcal{T}_{h}\right\}_{h}$ of $\Omega$. Moreover, for $\theta \in[0,1 / 2)$, assume that the following restriction holds on the time step:

$$
\Delta t<\frac{2 \alpha^{a} h^{2}}{(1-2 \theta)\left(M^{a}\right)^{2} \widetilde{C}_{0}^{2}},
$$

where $\alpha^{a}$ and $M^{a}$, given in equations (3.41) and (3.51), are the coercivity and continuity constants of the bilinear form $a_{h}(\cdot ; \cdot, \cdot)$ of equation (3.5), whereas $\widetilde{C}_{0}$ is given in (3.31). Then, under the hypotheses of Lemmas 3.9 and $3.10, u_{h}^{n}$ satisfies the following estimate for any $\theta \in[0,1]$ :

$$
\begin{aligned}
\left\|u_{h}^{n}\right\|_{\mathrm{L}^{2}(\Omega)} \leq & C_{\alpha^{a}, \widetilde{C}_{0}, T}\left[\left\|u_{h}^{0}\right\|_{\mathrm{L}^{2}(\Omega)}+M_{\boldsymbol{\sigma}, \boldsymbol{\beta}, \kappa, \Omega}^{(1)}\left(\|G\|_{\mathrm{L}^{\infty}\left(0, T ; \mathrm{L}^{2}(\Gamma)\right)}+\|f\|_{\mathrm{L}^{\infty}\left(0, T ; \mathrm{L}^{2}(\Omega)\right)}\right)\right. \\
& \left.+\left(M_{\boldsymbol{\beta}}+\frac{1}{\sqrt{h}} M_{\boldsymbol{\sigma}, \boldsymbol{\beta}, \kappa, \Omega}^{(2)}\right)\|g\|_{\mathrm{L}^{\infty}\left(0, T ; \mathrm{L}^{2}(\Gamma)\right)}\right],
\end{aligned}
$$

where $C_{\alpha^{a}, \widetilde{C}_{0}, T}$ is a positive constant depending on $\alpha^{a}, \widetilde{C}_{0}$, and $T$, but independent of $N, \Delta t$, and $h$. Moreover, $M_{\boldsymbol{\sigma}, \boldsymbol{\beta}, \kappa, \Omega}^{(1)}, M_{\boldsymbol{\beta}}$, and $M_{\boldsymbol{\sigma}, \boldsymbol{\beta}, \kappa, \Omega}^{(2)}$ are positive constants depending on the domain $\Omega$ and the data of the problem, reading:

$$
\begin{aligned}
& M_{\boldsymbol{\sigma}, \boldsymbol{\beta}, \kappa, \Omega}^{(1)}:=\max \left\{\frac{\sqrt{2}}{C_{\Omega}}, \frac{\sqrt{2} C_{\mathrm{tr}}}{C_{\Omega}}+\frac{1}{|\boldsymbol{\sigma}|} \sqrt{\frac{C_{\Omega_{B}}}{\bar{\xi}_{0}}}\left(\|\boldsymbol{\sigma}\|_{\mathrm{W}^{1, \infty}\left(Q_{T}\right)} \sqrt{\bar{C}_{2}}+\|\boldsymbol{\beta} \cdot \mathbf{n}\|_{\mathrm{L}^{\infty}\left(Q_{T}\right)} \sqrt{\bar{C}_{1}}\right)\right\}, \\
& M_{\boldsymbol{\beta}}=\sqrt{2}\|\boldsymbol{\beta} \cdot \mathbf{n}\|_{\mathrm{L}^{\infty}\left(Q_{T}\right)}, \\
& M_{\boldsymbol{\sigma}, \boldsymbol{\beta}, \kappa, \Omega}^{(2)}:=2\|\boldsymbol{\sigma}\|_{\mathrm{W}^{1, \infty}\left(Q_{T}\right)} \sqrt{C_{\Omega_{B}} \bar{C}_{2}}+|\boldsymbol{\sigma}| \sqrt{\bar{\xi}_{\infty}} .
\end{aligned}
$$

Proof. Let us take $\varphi_{h}=u_{h, \theta}^{n}$ in equation (3.55), then, for any $n \geq 1$, we have:

$$
\frac{1}{2}\left(\left\|u_{h}^{n}\right\|_{L^{2}(\Omega)}^{2}-\left\|u_{h}^{n-1}\right\|_{L^{2}(\Omega)}^{2}\right)+\left(\theta-\frac{1}{2}\right)\left\|u_{h}^{n}-u_{h}^{n-1}\right\|_{L^{2}(\Omega)}^{2}+\Delta t a_{h}\left(t_{\theta}^{n} ; u_{h, \theta}^{n}, u_{h, \theta}^{n}\right)=\Delta t \mathcal{F}_{h}\left(t_{\theta}^{n} ; u_{h, \theta}^{n}\right) .
$$

By the coercivity of the bilinear form $a_{h}(\cdot ; \cdot, \cdot)$ of Lemma 3.9 and by using the continuity constant $M_{h}^{\mathcal{F}}$ of equation $(3.53)$ for the functional $\mathcal{F}_{h}(\cdot ; \cdot)$, we get, for any $n \geq 1$ :

$$
\left\|u_{h}^{n}\right\|_{\mathrm{L}^{2}(\Omega)}^{2}-\left\|u_{h}^{n-1}\right\|_{\mathrm{L}^{2}(\Omega)}^{2}+(2 \theta-1)\left\|u_{h}^{n}-u_{h}^{n-1}\right\|_{\mathrm{L}^{2}(\Omega)}^{2}+2 \Delta t \alpha^{a}\left\|u_{h, \theta}^{n}\right\|_{h}^{2} \leq 2 \Delta t M_{h}^{\mathcal{F}}\left\|u_{h, \theta}^{n}\right\|_{h} .
$$

By using a Young's type inequality, we have for some $\epsilon>0$ :

$$
\left\|u_{h}^{n}\right\|_{L^{2}(\Omega)}^{2}-\left\|u_{h}^{n-1}\right\|_{L^{2}(\Omega)}^{2}+(2 \theta-1)\left\|u_{h}^{n}-u_{h}^{n-1}\right\|_{L^{2}(\Omega)}^{2}+\Delta t(2-\epsilon) \alpha^{a}\left\|u_{h, \theta}^{n}\right\|_{h}^{2} \leq \Delta t \frac{\left(M_{h}^{\mathcal{F}}\right)^{2}}{\epsilon \alpha^{a}}
$$

If $\theta \in[1 / 2,1]$, by setting $\epsilon=2$, the left hand side of (3.60) can be bounded as:

$$
\left\|u_{h}^{n}\right\|_{L^{2}(\Omega)}^{2}-\left\|u_{h}^{n-1}\right\|_{L^{2}(\Omega)}^{2} \leq \Delta t \frac{\left(M_{h}^{\mathcal{F}}\right)^{2}}{2 \alpha^{a}} .
$$


If $\theta \in[0,1 / 2)$, we start by considering in equation (3.55) $\varphi_{h}=u_{h}^{n}-u_{h}^{n-1}$; then, we have:

$$
\left\|u_{h}^{n}-u_{h}^{n-1}\right\|_{L^{2}(\Omega)}^{2}=-\Delta t a_{h}\left(t_{\theta}^{n} ; u_{h, \theta}^{n}, u_{h}^{n}-u_{h}^{n-1}\right)+\Delta t \mathcal{F}_{h}\left(t_{\theta}^{n} ; u_{h}^{n}-u_{h}^{n-1}\right) .
$$

By using Lemmas 3.7, 3.9, and 3.10, we obtain:

$$
\begin{aligned}
\left\|u_{h}^{n}-u_{h}^{n-1}\right\|_{\mathrm{L}^{2}(\Omega)}^{2} & \leq \Delta t M^{a}\left\|u_{h, \theta}^{n}\right\|_{h}\left\|u_{h}^{n}-u_{h}^{n-1}\right\|_{h}+\Delta t M_{h}^{\mathcal{F}}\left\|u_{h}^{n}-u_{h}^{n-1}\right\|_{h} \\
& \leq \Delta t M^{a}\left\|u_{h, \theta}^{n}\right\|_{h} \frac{\widetilde{C}_{0}}{h}\left\|u_{h}^{n}-u_{h}^{n-1}\right\|_{L^{2}(\Omega)}+\Delta t M_{h}^{\mathcal{F}} \frac{\widetilde{C}_{0}}{h}\left\|u_{h}^{n}-u_{h}^{n-1}\right\|_{L^{2}(\Omega)} .
\end{aligned}
$$

Therefore, the left hand side of (3.60) can be bounded from below as:

$$
\begin{aligned}
\left\|u_{h}^{n}\right\|_{L^{2}(\Omega)}^{2}-\left\|u_{h}^{n-1}\right\|_{L^{2}(\Omega)}^{2}+(2 \theta-1) \Delta t^{2} \frac{\widetilde{C}_{0}^{2}}{h^{2}}\left(\left(M^{a}\right)^{2}\right. & \left\|u_{h, \theta}^{n}\right\|_{h}^{2}+\left(M_{h}^{\mathcal{F}}\right)^{2} \\
& \left.+2 M^{a} M_{h}^{\mathcal{F}}\left\|u_{h, \theta}^{n}\right\|_{h}\right)+\Delta t(2-\epsilon) \alpha^{a}\left\|u_{h, \theta}^{n}\right\|_{h}^{2} \leq \Delta t \frac{\left(M_{h}^{\mathcal{F}}\right)^{2}}{\epsilon \alpha^{a}}
\end{aligned}
$$

for some $\epsilon>0$. By rearranging terms, we finally obtain:

$$
\begin{aligned}
\left\|u_{h}^{n}\right\|_{L^{2}(\Omega)}^{2} & -\left\|u_{h}^{n-1}\right\|_{L^{2}(\Omega)}^{2}+\Delta t\left\|u_{h, \theta}^{n}\right\|_{h}\left((2-\epsilon) \alpha^{a}-(1-2 \theta) \Delta t\left(M^{a}\right)^{2} \frac{\widetilde{C}_{0}^{2}}{h^{2}}\right) \\
& \leq\left(\frac{\Delta t}{\epsilon \alpha^{a}}+(1-2 \theta) \Delta t^{2} \frac{\widetilde{C}_{0}^{2}}{h^{2}}\right)\left(M_{h}^{\mathcal{F}}\right)^{2}-2 \Delta t^{2} M^{a} M_{h}^{\mathcal{F}}\left\|u_{h, \theta}^{n}\right\|_{h} \frac{\widetilde{C}_{0}^{2}}{h^{2}} \\
& \leq \Delta t \max \left\{\frac{1}{\epsilon \alpha^{a}},(1-2 \theta) \widetilde{C}_{0}^{2}\right\}\left(1+\Delta t \frac{1}{h^{2}}\right)\left(M_{h}^{\mathcal{F}}\right)^{2} .
\end{aligned}
$$

Under the hypothesis of equation (3.57) and by choosing $\epsilon>\frac{1-2 \theta}{2 \alpha^{a}}>0$ we obtain:

$$
\left\|u_{h}^{n}\right\|_{\mathrm{L}^{2}(\Omega)}^{2}-\left\|u_{h}^{n-1}\right\|_{\mathrm{L}^{2}(\Omega)}^{2} \leq C_{\epsilon, \alpha^{a}, \widetilde{C}_{0}} \Delta t\left(M_{h}^{\mathcal{F}}\right)^{2},
$$

with $C_{\epsilon, \alpha^{a}, \widetilde{C}_{0}}:=\Delta t \max \left\{\frac{1}{\epsilon \alpha^{a}},(1-2 \theta) \widetilde{C}_{0}^{2}\right\}\left(1+\Delta t \frac{1}{h^{2}}\right)$.

Therefore, for any $\theta \in[0,1]$ and any fixed integer $m$ such that $1 \leq m \leq N_{T}$, we obtain:

$$
\left\|u_{h}^{m}\right\|_{\mathrm{L}^{2}(\Omega)}^{2} \leq\left\|u_{h}^{0}\right\|_{\mathrm{L}^{2}(\Omega)}^{2}+C_{\alpha^{a}, \widetilde{C}_{0}} \Delta t \sum_{n=0}^{m-1}\left(M_{h}^{\mathcal{F}}\right)^{2} \leq\left(\left\|u_{h}^{0}\right\|_{\mathrm{L}^{2}(\Omega)}+\sqrt{C_{\alpha^{a}, \widetilde{C}_{0}} m \Delta t} M_{h}^{\mathcal{F}}\right)^{2},
$$

with $C_{\alpha^{a}, \widetilde{C}_{0}}$ a constant depending on $\frac{1}{\alpha^{a}}$ and $\widetilde{C}_{0}$. The thesis follows by the expression of $M_{h}^{\mathcal{F}}$ of (3.53) by considering $C_{\alpha^{a}, \widetilde{C}_{0}, T}:=\sqrt{C_{\alpha^{a}, \widetilde{C}_{0}} T}$.

We notice that the last term of the bound (3.58) tends to $+\infty$ as the mesh size $h$ tends to zero, due to the dependency of $M_{h}^{\mathcal{F}}$ on $h$. Therefore, the result of Theorem 3.13 does not imply the stability of the solution with respect to $h$. Indeed, the finer the mesh size, the larger is the growth of the constant in the a priori estimate. This result is coherent with that of [27]; as a matter of fact the weak imposition of the Dirichlet BCs represents a particular case of equation (3.55), as already observed in Remark 3.1. However, as reported in Sections 4 and [31], stable (i.e. $h$ independent) numerical results are obtained also in this case.

Remark 3.14. The numerical tools and recipes introduced in Sections 2 and 3 can be suitably used to obtain a priori error estimates with respect to the spatial and time discretizations. The technical details for the derivations of such error estimates will be reported in [31]. 


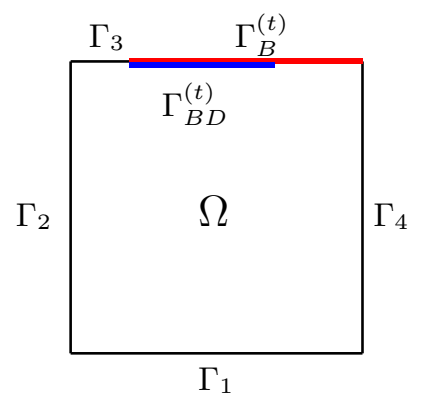

Figure 1. Computational domain: $\Omega=(0,1)^{2}$; the red line represents the part of the boundary, $\Gamma_{B}^{(t)}$, on which we set mixed time varying BCs, while $\Gamma_{B D}^{(t)} \subseteq \Gamma_{B}^{(t)}$, the blue one, its the subset where we impose Dirichlet BCs at time $t \in(0, T)$. (Color online).

\section{A NUMERICAL EXAMPLE}

We solve, by the proposed method, the heat equation complemented with mixed time varying BCs. Our example considers an elliptic operator involving only the diffusive term. We compare the solutions obtained to the the standard time varying FE method's solutions and we refer the reader to [31] for a more exhaustive discussion on numerical results.

By referring to equation (2.6), we consider a problem defined for $t \in(0, T)$, with $T=3$, in the domain $\Omega=(0,1)^{2}$ given in Figure 1 with boundary defined as in equation (2.4), where $\Gamma_{D}^{(t)}=\Gamma_{1} \cup \Gamma_{2} \cup \Gamma_{3} \cup \Gamma_{B D}^{(t)} \cup \Gamma_{4}$; $\Gamma_{N}^{(t)}:=\Gamma_{B}^{(t)} \backslash \Gamma_{B D}^{(t)}$ represents the Neumann boundary. Specifically, the subset of the boundary $\Gamma_{B D}^{(t)} \subseteq \Gamma_{B}^{(t)}$ is defined as:

$$
\Gamma_{B D}^{(t)}:=\left\{(x, y) \in \mathbb{R}^{2}: x \in I^{(t)}, y=1\right\}, \text { with } I^{(t)}:= \begin{cases}\emptyset & \text { for } t<0.2 \text { and } 1.5 \leq t<3 \\ (-2 t+1.4,1) & \text { for } 0.2 \leq t<0.6, \\ (0.2,1) & \text { for } 0.6 \leq t<1, \\ (1.6 t-1.4,1) & \text { for } 1 \leq t<1.5 .\end{cases}
$$

As $\Sigma=\Gamma \times(0, T)$ satisfies the hypotheses of Theorem 2.1, the continuous problem is well-posed. On $\Sigma_{D}$ and $\Sigma_{N}$, we prescribe homogeneous Dirichlet and Neumann BCs as in equations (2.5) by setting $g=0$ on $\Sigma_{D}$ and $G=0$ on $\Sigma_{N}$. The initial condition is $u_{0}=0$ in $\Omega$ and the source term is defined as $f=1$ in $Q_{T}$. Finally, as for the linear second order elliptic operator $\mathcal{L}$ of equation (2.2), we consider the diffusivity tensor to be isotropic, i.e. $\boldsymbol{\sigma}=\sigma \boldsymbol{I}$, with $\boldsymbol{I}$ the identity tensor and $\sigma=0.1$; moreover, $\boldsymbol{\beta}=\mathbf{0}$ and $\kappa=0$.

We use Lagrange $\mathbb{P}_{1}$ finite elements for the discretization in space, i.e. we consider in equations (2.14) and (3.3), $\mathcal{V}_{h}^{n}$ and $\mathcal{V}_{h}$ defined for degree $k=1$, respectively, and the BE method ( $\theta=1$ in the general $\theta$-scheme) for the time discretization, with time step $\Delta t=0.01$. The problem approximated by using the time varying FE method described in Section 2.2 has been implemented in FreeFem++ [16], for which the considerations of Remark 2.3 apply. Similarly, the full discrete problem (3.55) complemented with mixed time varying BCs has been implemented in FreeFem++. Specifically, following Remarks 3.1 and 3.2, we set the values of the functions $\gamma(\mathbf{x}, t)$ and $\xi(\mathbf{x}, t)$ as:

$$
\gamma(\mathbf{x}, t):=\left\{\begin{array}{ll}
10^{-8} & \text { for } \mathbf{x} \in \Gamma_{N}^{(t)}, \\
10^{4} & \text { for } \mathbf{x} \in \Gamma_{D}^{(t)}
\end{array} \quad \text { and } \quad \xi(\mathbf{x}, t):=\left\{\begin{array}{ll}
10^{1} & \text { for } \mathbf{x} \in \Gamma_{N}^{(t)}, \\
10^{8} & \text { for } \mathbf{x} \in \Gamma_{D}^{(t)},
\end{array} \quad \forall t \in(0, T)\right.\right.
$$

respectively. Following this choice, we have that the lower bound $\bar{\xi}_{0}=10$ is larger than the right hand side of condition (3.40); indeed, we computationally estimate the latter to be about 1.5. 


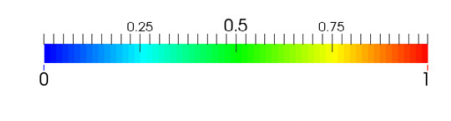

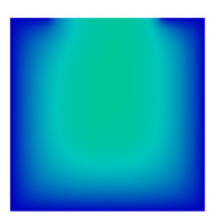

(a) $\quad t=0.3$

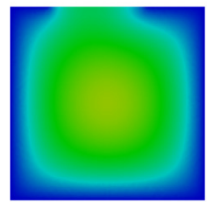

(a) $t=1.3$

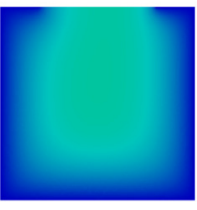

(b)

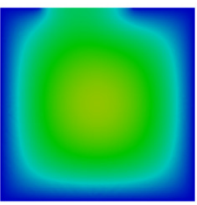

(b)

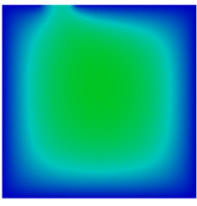

(a) $t=0.5$

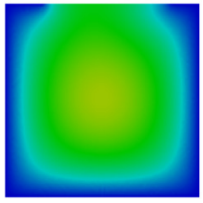

(a) $t=1.4$

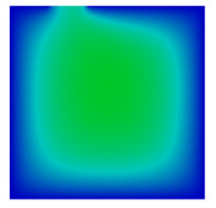

(b)

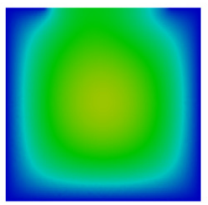

(b)
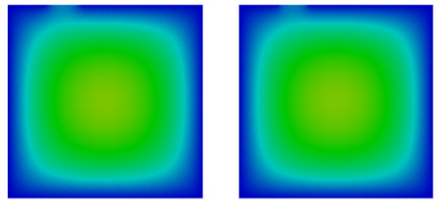

(a) $\quad t=1.1$

(b)

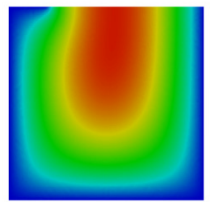

(a) $t=3.0$

(b)

Figure 2. Solutions at different time steps for $h=0.00625$; comparison between the Nitsche's method (case (a)) and the time varying FE method (case (b)) for some $t \in(0,3)$.

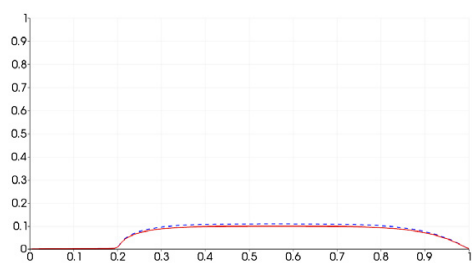

(A) $t=0.1$

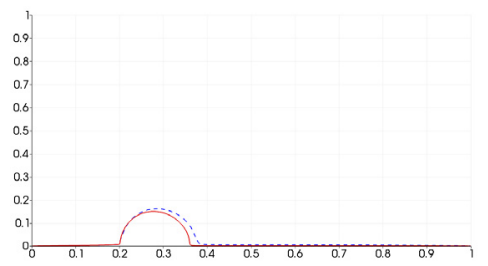

(D) $t=1.1$

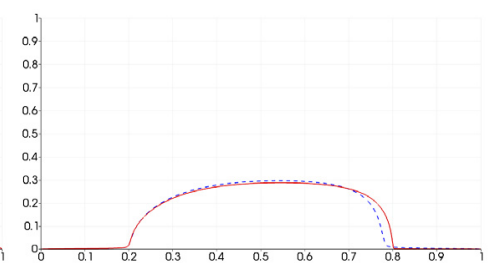

(B) $t=0.3$.

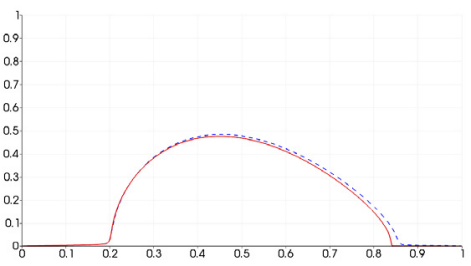

(E) $t=1.4$.

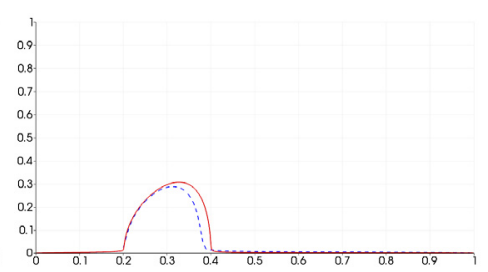

(c) $t=0.5$.

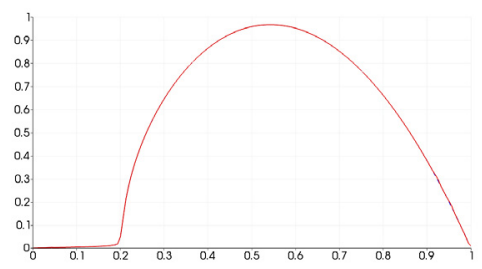

(F) $t=3$.

Figure 3. Solutions on $\Gamma_{3} \cup \Gamma_{B}^{(t)}$ at different time steps by considering the mesh size $h=$ 0.00625; comparison between the Nitsche's method (- -) and the time varying FE method (-) solutions for some $t \in(0,3)$.

In Figure 2, we report the evolution of the computed solution at different time steps by considering the mesh size $h=0.00625$. By a qualitative comparison between the Nitsche's method (case (a)) and the time varying FE method (case (b)) the solutions look qualitatively very similar. In Figure 3, we compare the solutions profile computed at different time steps through the upper bound of the domain $\Gamma_{3} \cup \Gamma_{B}^{(t)}$, by considering the mesh size $h=0.00625$. We notice that the solutions obtained by using the Nitsche's method (-) result in a smoother curve, especially near the boundary between $\Sigma_{D}$ and $\Sigma_{N}$, with respect to the profile of the approximate solution computed with the time varying FE method (-). This is in agreement with the results obtained in [6] for the linear advection-diffusion equation, where the authors observe that the weak imposition of the Dirichlet BCs 


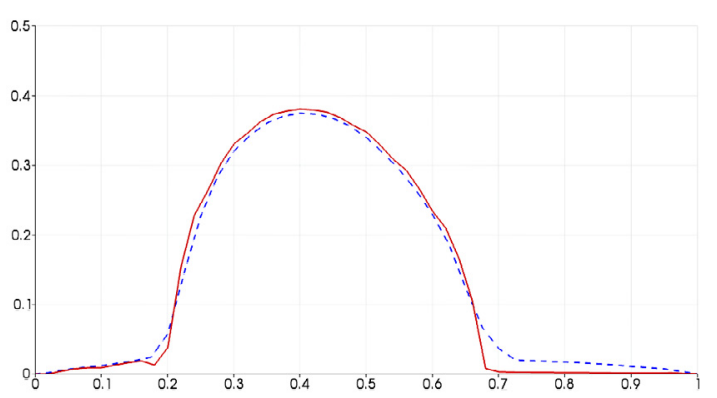

(A) $h=0.025$.

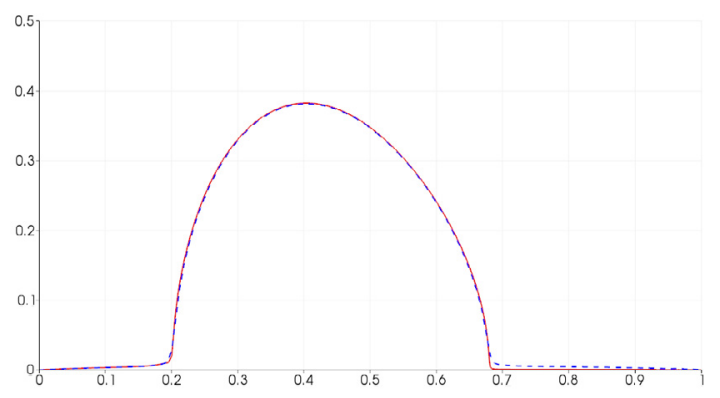

(c) $h=0.00625$.

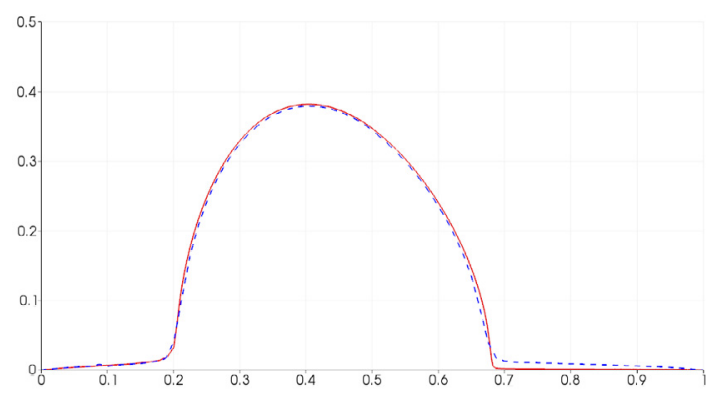

(в) $h=0.0125$.

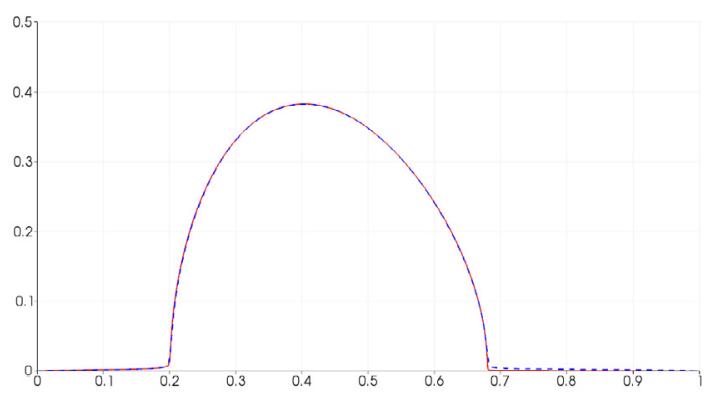

(D) $h=0.003125$.

FiguRE 4 . Solutions on $\Gamma_{3} \cup \Gamma_{B}^{(t)}$ at $t=1.3$ by considering different mesh sizes (h); comparison between the Nitsche's method (--) and the time varying FE method $(-)$ solutions.

TABLE 1. Comparison of the results (CPU time, number of elements, and DoFs) obtained by using the Nitsche's method and the time varying FE method for different characteristic mesh sizes $(h)$.

\begin{tabular}{lcccccccc}
\hline & \multicolumn{3}{c}{ Nitsche's method } & \multicolumn{5}{c}{ time varying FE method } \\
\cline { 2 - 8 }$h$ & $N_{e l}$ & $N_{\text {DoFs }}$ & CPU Time (s) & $\min N_{e l}$ & $\max N_{e l}$ & $\min N_{\text {DoFs }}$ & $\max N_{\text {DoFs }}$ & CPU Time (s) \\
\hline 0.025 & 3808 & 1985 & 22 & 3814 & 10208 & 1988 & 5249 & 48 \\
0.0125 & 15176 & 7749 & 95 & 15094 & 40740 & 7708 & 20659 & 208 \\
0.00625 & 60594 & 30618 & 409 & 60624 & 164294 & 30633 & 82724 & 887 \\
0.003125 & 243178 & 122230 & 2200 & 242642 & 657158 & 121962 & 329732 & 4443 \\
\hline
\end{tabular}

is able to reduce numerical oscillations, which may be caused by discontinuous boundary data. In Figure 4 , we compare the solutions profile computed at $t=1.3$ through $\Gamma_{3} \cup \Gamma_{B}^{(t)}$, by considering different mesh sizes $h=0.025,0.0125,0.00625$, and 0.003125 . From the comparison between the Nitsche's method (-) and the time varying FE method (-), we can observe that the proposed method shows qualitatively good solutions already with a coarse mesh, thus indirectly showing the effectiveness of the Nitsche's method.

In Table 1, we report, for different values of the mesh size $h=0.025,0.0125,0.00625$, and 0.003125 , the number of elements $\left(N_{e l}\right)$ and the degrees of freedom $\left(N_{\text {DoFs }}\right)$ associated to the FE spaces $\mathcal{V}_{h}$ for the Nitsche's method, and the minimum $(\min )$ and maximum $(\max )$ number of elements and degrees of freedom associated to the time varying FE spaces $\mathcal{V}_{h}^{n}$, respectively. Moreover, we record the corresponding CPU times. The smaller the mesh size, the larger are the saving of the computational costs allowed by the Nitsche's method with respect 


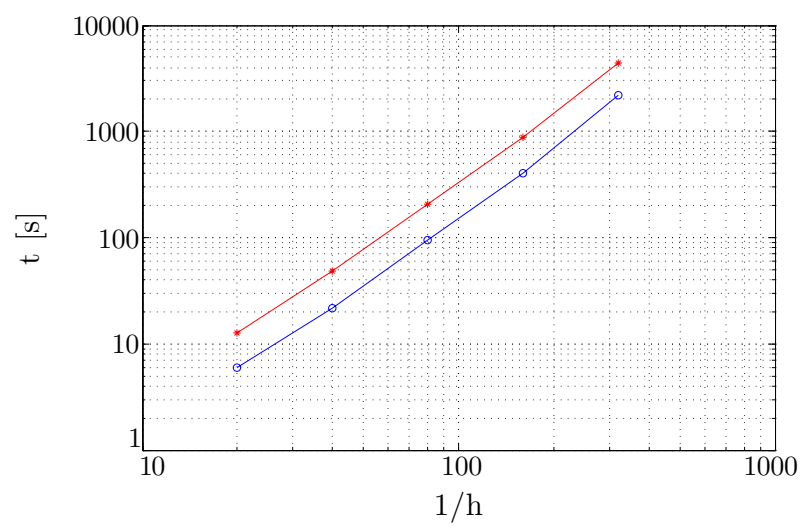

Figure 5. Mesh size $(1 / h)$ vs. CPU time in seconds; comparison between the Nitsche's method $(-)$ and the time varying FE method (-) solutions.

to the time varying FE method, as highlighted in Figure 5; indeed, for $h=0.003125$, the number of DoFs is about the half with the proposed Nitsche's method.

\section{Conclusions}

In this work, we considered a numerical approach developed within the FE method to solve initial boundary value problems associated with parabolic second order PDEs endowed with mixed time varying BCs. Specifically, we proposed a numerical scheme based on the Nitsche's method in the framework of [17], but by considering the more general case of time varying coefficients. We proved the existence and the numerical stability of the solution based on the $\theta$-method for the discretization in time; as expected, we obtained a stability condition of the full discrete scheme dependent on the mesh size, according with the theory developed in the framework of the weak imposition of the Dirichlet BCs [27]. Moreover, we presented some numerical results which highlight the performance and, specifically, the computational efficiency of the proposed full discrete scheme compared to a standard time varying FE method based on remeshing strategies, which represents the typical approach for problems with mixed time varying BCs.

\section{REFERENCES}

[1] P. Acquistapace and B. Terreni, Linear parabolic equations in Banach spaces with variable domains but constant interpolation spaces. Ann. Sc. Norm. Super. Pisa 13 (1986) 75-107.

[2] R.A. Adams and J.J.F. Fournier, Sobolev Spaces. Academic Press, New York (2003).

[3] D.N. Arnold, An interior penalty Finite Element method with discontinuous elements. SIAM J. Numer. Anal. 19 (1982) $742-760$.

[4] I. Babuška, The Finite Element method with Lagrangian multipliers. Numer. Math. 20 (1973) 179-192.

[5] C. Baiocchi, Problemi misti per l'equazione del calore. Rendiconti del Seminario Matematico e Fisico di Milano. Università Studi Milano, XLI, Milan (1971) 3-38.

[6] Y. Bazilevs and T.J.R. Hughes, Weak imposition of Dirichlet boundary conditions in fluid mechanics. Comput. Fluids 36 (2007) 12-26.

[7] Y. Bazilevs, C. Michler, V.M. Calo, and T.J.R. Hughes, Weak Dirichlet boundary conditions for wall-bounded turbulent flows. Comput. Methods Appl. Mech. Eng. 196 (2007) 4853-4862.

[8] J. Bergh and J. Löfström, Interpolation Spaces. An Introduction. Springer-Verlag, Berlin, Heidelberg, New York (1976).

[9] A. Bove, B. Franchi, and E. Obrecht, Parabolic problems with mixed time dependent lateral conditions. Commun. Partial Differ. Equ. 7 (1982) 134-156.

[10] A. Bove, B. Franchi and E. Obrecht, Boundary value problems with mixed lateral conditions for parabolic operators. Ann. Mat. Pura Appl. 131 (1982) 375-413.

[11] F. Brezzi and M. Fortin, Mixed and Hybrid Finite Element Methods. Springer-Verlag, New York (1991).

[12] J. Butcher, The Numerical Analysis of Ordinary Differential Equations. Wiley, New York (1987). 
[13] P.G. Ciarlet, The Finite Element Method for Elliptic Problems. North-Holland, Amsterdam (1978).

[14] C. Dawson, S. Sun, and M. Wheeler, Compatible algorithms for coupled flow and transport. Comput. Methods Appl. Mech. Eng. 193 (2004) 2565-2580.

[15] A. Ern and J.L. Guermond, Theory and Practice of Finite Elements. Springer-Verlag, New York (2004).

[16] F. Hecht, New development in FreeFem++. J. Numer. Math. 20 (2012) 251-265.

[17] M. Juntunen and R. Stenberg, Nitsche's method for general boundary conditions. Math. Comput. 78 (2009) $1353-1374$.

[18] T. Kato, Abstract evolution equations of parabolic type in Banach and Hilbert spaces. Nagoya Math. J. 19 (1961) 93-125.

[19] J.L. Lions and E. Magenes, Non Homogeneous Boundary Value Problems and Applications. Vol. I, II. Springer-Verlag, Berlin (1972).

[20] J.L. Lions and J. Peetre, Sur une classe d'espaces d'interpolation. Publ. Math. Inst. Hautes Étud. Sci. 19 (1964) 5-68.

[21] E. Magenes, Problemi al contorno misti per I'equazione del calore. Rend. Semin. Mat. Univ. Padova 24 (1955) 1-28.

[22] J. Nitsche, Über ein Variationsprinzip zur Lösung von Dirichlet-Problemen bei Verwendung von Teilräumen, die keinen Randbedingungen unterworfen sind. Abh. Math. Semin. Univ. 36 (1971) 9-15.

[23] F. Nobile and C. Vergara, An effective fluid-structure interaction formulation for vascular dynamics by generalized Robin conditions. SIAM J. Scientific Comput. 30 (2008) 731-763.

[24] J.T. Oden, I. Babǔska, and C. Baumann, A discontinuous hp finite element method for diffusion problems. J. Comput. Phys. 146 (1998) 491-519.

[25] A. Quarteroni, Numerical Models for Differential Problems. Springer-Verlag, Milan (2014).

[26] A. Quarteroni and A. Valli, Numerical Approximation of Partial Differential Equations. Springer-Verlag, Berlin, Heidelberg (1994).

[27] B. Rivière, Discontinuous Galerkin Methods For Solving Elliptic and Parabolic Equations: Theory and Implementation. SIAM, Philadelphia, PA, USA (2008).

[28] B. Rivière, M.F. Wheeler, and V. Girault, A priori error estimates for finite element methods based on discontinuous approximation spaces for elliptic problems. SIAM J. Numer. Anal. 39 (2001) 902-931.

[29] G. Savaré, Parabolic problems with mixed variable lateral conditions: an abstract approach. J. Pure Appl. Math. 76 (1997) 321-351.

[30] S. Sun and M.F. Wheeler, Symmetric and nonsymmetric discontinuous Galerkin methods for reactive transport in porous media. SIAM J. Numer. Anal. 43 (2005) 195-219.

[31] A. Tagliabue, Ph.D. thesis, Politecnico di Milano, Italy. In preparation (2016).

[32] R. Temam, Navier-Stokes Equations. Theory and Numerical Results. North-Holland Publishing Company, Amsterdam, New York-Oxford (1977).

[33] V. Thomée, Galerkin Finite Element Methods for Parabolic Problems. Springer Verlag, Berlin, Heidelberg (1984).

[34] C. Vergara, Nitsche's method for defective boundary value problems in incompressible fluid-dynamics. J. Sci. Comput. 46 (2011) 100-123.

[35] M.F. Wheeler, An elliptic collocation-finite element method with interior penalties. SIAM J. Numer. Anal. 15 (1978) $152-161$. 